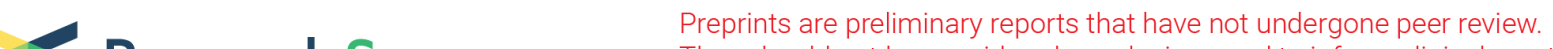 Research Square
or referenced by the media as validated information.
}

\section{In Vivo and in Vitro Evaluation of the Anti- inflammatory Effect of Thymoquinone Toward Ischemic Brain Injury Alleviation via Nrf2/HO-1 Pathway}

\section{Nashwa Amin}

zhejiang university,School of medicine

\section{Xiaoxue Du}

Zhejiang Provincial First Hospital: Zhejiang University School of Medicine First Affiliated Hospital

\section{Shijia Chen}

zhejiang university,school of medicine

\section{Qiannan Ren}

Zhejiang Medical University: Zhejiang University School of Medicine

\section{Azhar Badry}

zhejiang university school of medicine

\section{Benson O.A. Botchway}

Zhejiang University School of Medicine

\section{Zhiying Hu}

Zhejiang University of Traditional Chinese Medicine Affiliated Integrated Chinese and Western Medicine Hospital: Hangzhou Red Cross Hospital

\section{Marong Fang ( $\nabla$ fangmaro@zju.edu.cn )}

Institute of Neuroscience, School of Medicine, Zhejiang University, Hangzhou, China.

https://orcid.org/0000-0002-6636-4347

\section{Research}

Keywords: Cerebral Ischemia, Thymoquinone (TQ), Inflammation, Nrf2/HO-1, Oxidative Stress, PC12, $\mathrm{OGD} / \mathrm{R}$

Posted Date: October 22nd, 2020

DOI: https://doi.org/10.21203/rs.3.rs-93479/v1

License: (c) (1) This work is licensed under a Creative Commons Attribution 4.0 International License. Read Full License 


\section{Abstract}

Background - In recent years, considerable efforts have been devoted to exploring effective therapy for cerebral ischemia. Reactive oxygen species (ROS) mediated - inflammation plays a crucial role in ischemic brain injury. Triptolide (TP) has been widely used for ischemic therapy although administrating a chronic dose of this therapy may cause serious drawbacks and higher liver toxicity. Considering these critical side effects, here we demonstrate the employment of thymoquinone (TQ) as a new alternative drug for alleviating ischemic brain damage via suppression of inflammatory cytokines by inducing Nrf2/HO-1 under a chronic dose without toxicity.

Methods-We assessed a photo-thrombosis mouse model of focal cerebral ischemia to investigate the impact of the chronic dose of TQ to alleviates ischemic brain damage, meanwhile, we used Pc12 to determine the efficiency of TQ to attenuate the OGD/R induces cell death.

Results- Our in vivo and in vitro results indicate that the administration of TQ drug can sufficiently mitigate the brain damage after stroke by increasing the Nrf2/HO-1 expression and thereby modulate the cell death and inflammation resulting from cerebral ischemia. The observation based on YFP mice elucidates the role of TQ therapy in recovering the brain status after injury through increasing the dendrite spines density and the ratio of YFP reporter cells with NeuN expression.

Conclusions- Our study is the first to focus on the crucial role of the Nrf2/HO-1 pathway as a promising ischemic therapy under a chronic dose of $\mathrm{TQ}$ by increasing proliferating protein expression, decreasing inflammation and neuronal cell death as well as controlling the autophagy process.

\section{Introduction}

Stroke has been considered as one the most common cause of death in developed countries [1]. The ischemic stroke, which results from the sudden blockage of blood supply to brain areas represents about $87 \%$ of stroke [2]. Such blockage may lead to brain dysfunction and injury owing to the insufficient supply of glucose and oxygen to brain cells [3]. Despite the recent therapeutic advances, ischemic stroke is still a crucial issue with a massive socioeconomic impact worldwide [4]. Several previous studies declared that neuroinflammation could largely induce the regulation and pathogenic progression of stroke injury as well as many neurodegenerative disorders such as Alzheimer's disease [5]. Besides, oxidative stress can damage the biological system and robustly correlates with inflammation [6]. Recently, plant extract and natural agents have been attracted to growing attention due to their capabilities to suppress free radicalmediated injuries with a slight side effect. Triptolide (TP) is a traditional Chinese medicine that can be obtained from the Tripterygium wilfordii plant [7]. Many reports demonstrated that TP possesses numerous side effects and toxicology properties [8]. On the other hand, a closer investigation of Thymoquinone (TQ) treatment extracted from the Nigella sativa plant displayed multiple therapeutic effects which can be applied in clinical research with exceptional therapeutic potential and no toxicity properties, particularly in combination with chemotherapeutic drugs [9]. Introduced results elucidated the 
relation between TQ drug effect and the major regulator of endogenous defense mechanisms in the body named as Nrf2 (nuclear erythroid 2 related factor 2) [10]. Moreover, the activation of Nrf2 was strongly associated with anti-inflammatory activity through NF-KB mechanisms [10]. Up to now, the employment of TQ as an ischemic stroke therapy still needs more focus. Motivated by these considerations, the present study investigates the influences of TQ and TP drugs on ischemic stroke (Supplementary Fig. I). The analyzed results indicate that the TQ drug possesses an efficient role to alleviate brain damage after stroke through decreasing neuronal cell death and inflammation. Additionally, the TQ effect mainly occurs by downregulating autophagy marker LC3/Atg5/Beclin-1, which is strongly upregulated after stroke. Besides, the pathway of TQ therapy and accompanied proteins are well addressed.

\section{Materials And Methods}

\section{Animal groups and drug administration}

Both Mice C57BL/6J and thy1-YFP-16 transgenic mice (average weight of $27 \pm 2 \mathrm{~g}$ ) were randomly divided into six groups $n=20$, denoted as Normal, TQ, TP, Stroke, TQ+ Stroke, and TP+Stroke. The stroke model was conducted only on the last 3 groups. The Normal group was injected with a mixture of saline solution and DMSO 1\% (Thermofisher, 85,190 ) intraperitoneally at a dose of $0.2 \mathrm{~mL} / 20 \mathrm{~g}$ of body weight. TQ and TQ+ Stroke groups were injected intraperitoneally (I.P) with $5 \mathrm{mg} / \mathrm{kg}$ body weight daily for 5 days before implementing the model and 7 days after [11-13]. TQ (Sigma, 274666) was dissolved in DMSO at a concentration of $10 \mathrm{mg} / \mathrm{mL}$ and then diluted with normal saline to attain a final concentration of 0.5 $\mathrm{mg} / \mathrm{mL}$. TP and TP+ Stroke groups were injected intraperitoneally with $0.5 \mathrm{mg} / \mathrm{kg}, 24 \mathrm{~h}$ before establishing the model, and 7 days after [14-16]. TP (Med Chem Express, USA\#39836) was dissolved in DMSO at a concentration of $5 \mathrm{mg} / \mathrm{mL}$ and then diluted by normal saline to a final concentration of 0.05 $\mathrm{mg} / \mathrm{mL}$. Further information about the establishment of the YFP mice (Supplementary data section 1 \& Fig. II).

\section{Inducing focal cerebral ischemia}

Various stroke models were used to produce moderate-to-severe levels of ischemia [17]. Rose Bengal (RB) photoinduced thrombus was used to create core ischemia which locally reduces the blood flow, and this strategy is an alternative path to middle cerebral artery occlusion [17]. The RB photoinduced thrombus model simulates the human stroke where the blood flow drops to $10 \%$ and neuronal cell death takes place in the ischemic core [18-19]. As well as, due to the difficulties to keep the animal within a stereotaxic frame to access carotid arteries and the complications associated with collateral circulation, RB photoinduced model is found to be a good choice. $10 \mathrm{mg} / \mathrm{mL}$ RB dye (Sigma-Aldrich, \#R3877) solution dissolved in Phosphate-buffered saline (PBS) was injected intraperitoneally at a dose of $100 \mathrm{mg} / \mathrm{kg} .5$ min later, a light-emitting diode cold light source (OPLENIC, MIL- HAL 3001) was fixed above the target area which covers the right forelimb motor cortex ( $1.75 \mathrm{~mm}$ lateral to Bregma, $+0.5 \mathrm{~mm}$ left hemisphere) of the mice brain. To induce focal cerebral ischemia, the target area was illuminated for $15 \mathrm{~min}$ then the wound was sutured. 


\section{Survival rate}

The animals were monitored daily up to the 7th day of treatment for signing moribundity and mortality if any. The body weight of animals was recorded daily before and after treatment.

\section{Behavioral tests}

\section{Rotarod test}

The movement coordination and anti-fatigue ability of mice were determined by analyzing the ratio of staying time on the rotarod [20]. For more information, please see the supplementary data (section 2).

\section{Cylinder test (spontaneous forelimb use asymmetry test)}

To evaluate the spontaneous use of the forelimb, we investigated the cylinder test [21]. For more information, please see the supplementary data (section 2).

\section{Sunflower test}

This test was used to identify forelimb motor function [22]. Briefly, we examined the number of pieces of sunflower seeds remaining after a fixed time as previously described [23]. For more information, please see the supplementary data (section 2).

\section{Western blotting}

The total proteins of the cortex tissue were extracted with 1\% PMSF (Phenylmethanesulfonyl fluoride, Beyotime ST505) in $1 \mathrm{~mL}$ of ice-cold RIPA buffer, with added protease inhibitor cocktail EDTA-free and phosphatase inhibitors. After homogenizing and centrifuging at $12000 \mathrm{rpm}$ for $20 \mathrm{~min}$ at $4{ }^{\circ} \mathrm{C}$, supernatant proteins were preserved at $-80^{\circ} \mathrm{C}$. Sample concentrations were determined with BCA kits (KeyGEN, Nanjing, China). Further information about the antibodies and methodologies are provided in the supplementary data (Section 3).

\section{Immunofluorescence}

Mice were anesthetized as previously described in the literature [24], transcardially perfused with $50 \mathrm{~mL}$ $( \pm)$ of $0.9 \%$ saline to flush the vascular blood, and then perfused with $4 \%$ paraformaldehyde in $0.01 \mathrm{M}$ phosphate-buffered saline (PBS, pH 7.4). The sectioning and staining processes are addressed in the supplementary data (Section 4).

\section{Imaging and Dendritic spine analysis}

The bright-field images were captured using an Olympus BX61 microscope and fluorescence images were captured using an Olympus BX53 manual microscope (Olympus Instruments, Yokohama, Japan). Confocal images were captured using an Olympus FV1200 microscope (Olympus, Yokohama, Japan) 
with an $80 \mathrm{~m}$ working distance $63 \times / 1.4$-numerical aperture oil-immersion objective (see the supplementary data-section 5)

\section{2,3,5-Triphenyl tetrazolium chloride (TTC) and Histological staining}

TTC staining was performed on the 7th day of the experiment [25]. TTC staining process and calculation of infarct volume can be found in the supplementary data (Section 6). The most frequently routine histological stains HE and Nissl were employed to assess the temporal and spatial patterns of postischemic morphological alterations [26]. Sectioning and staining processes are addressed in the supplementary data (Section 7).

\section{Real-time PCR}

Real-time PCR was conducted to detect RNA expression levels of ATG5, c-FOS, c-MYC, iNOS and 5-HT in the cortex. Real-time PCR specific primers for mouse $\beta$-actin (internal control) were designed using Primer Express software. The Trizol (Invitrogen) extraction reagent was used to extract cortex RNA. All procedures were carried out according to the manufacturer's instructions. The concentration and purity of RNA samples were determined by Thermo Nanodrop 2000. One microgram of RNA from each sample was reverse transcribed into cDNA, according to the instructions of the DBI-2220 Bestar qPCR RT Kit. 2044 Bestar SYBR Green qPCR master mix. The reaction program for the PCR was as follows: $50{ }^{\circ} \mathrm{C}$ for 2 min; $95^{\circ} \mathrm{C}$ for $10 \mathrm{~min} ; 40$ cycles of $95^{\circ} \mathrm{C}$ for $5 \mathrm{~s}, 55^{\circ} \mathrm{C}$ for $30 \mathrm{~s}$, and $72{ }^{\circ} \mathrm{C}$ for the $30 \mathrm{~s}$. All mRNA expression levels were detected by RT-PCR. Results were analyzed using the Biorad CFX manager program, version 3.0. Each experiment was done three times.

\section{The primers used were as follows:}

$\beta$-actin Forward: 5'- CTGTCCCTGTATGCCTCTG -3'.

$\beta$-actin Reverse: 5'- ATGTCACGCACGATTTCC -3'.

ATG5 Forward: 5'- AACTGAAAGAGAAGCAGAACCA -3'.

ATG5 Reverse: 5'-TGTCTCATAACCTTCTGAAAGTGC -3'.

iNOS Forward: 5'-CACCTTGGAGTTCACCCAGT-3'

iNOS Reverse: 5'-ACCACTCGTACTTGGGATGC-3'

c-FOS Forward: 5'-GGGGACAGCCTTTCCTA-CTA-3'

c-FOS Reverse: 5'-CTGTCACCGTGGGGATAAAG-3'

c-MYC Forward: 5'-TCGCTGCTGTCCTCCGAGTCC-3'

c-MYC Reverse: 5'-GGTTTGCCTCTTCTCCACAGAC-3' 
5-HT Forward: 5'-CCATCAGCAAGGACCACGGCTA-3'

5-HT Reverse: 5'-CCCGTAGAGAACCAGCATGAGCAA-3'

\section{Cell line Groups and Establishment of OGD/R model}

\section{In vitro immunofluorescence staining}

Cells on the coverslips were fixed in a $4 \%$ paraformaldehyde for $1 \mathrm{hr}$. at room. The fixed cells were permeabilized with $0.2 \%$ Triton X-100 in $0.01 \mathrm{M}$ PBS for $15 \mathrm{~min}$. After blocking with $5 \%$ BSA in $0.01 \mathrm{M}$ PBST for $2 \mathrm{hr}$., the fixed cells were incubated with the primary antibody at $4^{\circ} \mathrm{C}$ overnight (Supplementary data-section 10).

\section{FACS analysis by using Annexin V/PI staining:}

Cells were washed twice with cold PBS and then resuspend in 1X Binding Buffer at a concentration of $1 \mathrm{x}$ $10^{\wedge} 6 \mathrm{cells} / \mathrm{ml}$. We transferred $100 \mu \mathrm{l}$ of the solution $\left(1 \times 10^{\wedge} 5\right.$ cells $)$ to a $5 \mathrm{ml}$ culture tube, then $5 \mu \mathrm{l}$ of FITC Annexin $\mathrm{V}$ and $5 \mu \mathrm{IPI}$ were added. cells were gently vortex and incubated for $15 \mathrm{~min}$ at $\mathrm{RT}\left(25^{\circ} \mathrm{C}\right)$ in the dark. Finally, we added $400 \mu \mathrm{l}$ of $1 \mathrm{X}$ Binding Buffer to each tube. Samples were analyzed by the CytoFLEX LX flow cytometer machine from Beckman within $1 \mathrm{~h}$. and data calculation by CytExpert software and significant values obtained by SPSS 20.0 .

\section{Statistical analysis}

Data were analyzed by one-way ANOVA using SPSS 20.0, followed by post hoc Fisher's least significant difference (LSD) tests. The $P$ values less than 0.05 were considered statistically significant. Histograms were generated in GraphPad Prism 5. All data are expressed as mean \pm SEM. Gray values of western blot results were calculated using Image Lab. Immunofluorescence and histological results were analyzed by

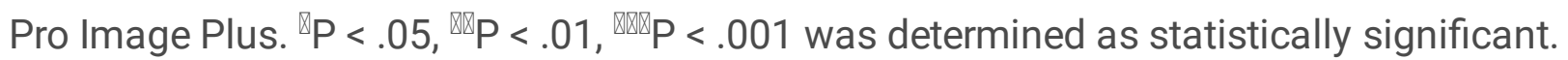

\section{Results}

\section{Effect of TQ drug on mice weight and Survival rate}

The observations of the mice weight and survival rate of all groups for 7 days shows a noticeable enhancement with TQ administration for 5 days before stroke as shown in Supplementary Fig. III (A\& B).

\section{TQ attenuate the motor dysfunction after stroke}

The pre-treatment of TQ for 5 days before model construction significantly improves the motor function compared with the stroke group $(P<0.01$, Fig. 1A). This elucidates that TQ injection for 5 days before establishing the stroke model affords more protective than TP injection. The forelimb motor function impairment and the ability of the mice to open seeds are investigated by using sunflower seed challenge. The obtained results show a lower ability to consume the sunflower seeds within the scheduled timeline 
for the stroke mice, while TQ administration can evoked this effect $(p<0.001)$ as shown in Fig. 1C, D. Findings obtained from the cylinder test (Fig.. 1B) demonstrate an enhancement in the impaired forelimb use in $\mathrm{TQ}+$ stroke mice at the $2^{\text {nd }}$ and $4^{\text {th }}$ day from the model $(P<0.05)$. Besides, a significant improvement in the impaired forelimb is observed on the $6^{\text {th }}$ day $(P<0.01)($ Fig. 1B), suggesting the high capability of TQ to attenuate the defect on the forelimb after stroke.

\section{TQ administration have no toxicity on liver tissue}

The influence of TQ and TP on the liver after 7 days from the injection can be observed by H\&E staining can be illustrated in Supplementary Fig. IV.

\section{TQ significantly reduce the infraction area after cerebral brain ischemia}

The analysis of TTC results in the mouse brain is presented in Fig. 2D. The stroke group reveals a significant infraction area $(\mathrm{P}<0.01)$ compared with the normal group (Fig. $2 \mathrm{E})$. The infraction area in the $\mathrm{TQ}+$ stroke group can be reduced noticeably compared with the stroke group $(\mathrm{P}<0.001)$. Besides, the histological changes within the injured area after stroke examined by Nissl and H\&E staining are shown in Fig. 2 A and B. After 7 days from applying the stroke model, a loss of Nissl substance in Nissl stains can be seen (Fig. 2B). The existence of acute neuronal degeneration after stroke is represented by red dead neurons. These neurons are characterized by darkly stained pyknotic nuclei, cell body shrinkage, and an intensely stained red eosinophilic cytoplasm (blue arrow). Such changes are frequently associated with vacuolar alteration within the cytoplasm and finely vacuolated adjacent tissue (red arrow) [27]. The changes may be altered by using TQ or TP administration. The number of neuron cells is usually used to indicate the brain-damaged area. Results declare that the number of neuron cells after stroke reduces $(P<0.01)$ (Fig. $2 C$ ), whereas the $T Q+S t r o k e$ group shows an increase in these neuron cells in neuron cell survival $(P<0.01)$ compared with the Stroke group.

\section{The effectiveness of TQ to attenuate the oxidative stress and cell death after brain injury}

Oxidative stress and apoptosis are major features in many neurodegenerative diseases. Therefore, the oxidative stress pathway NrF2/HO-1 (Fig. 3A-F) and the main apoptosis marker Bax/Bcl2/Caspase3 are investigated (Fig $4 \mathrm{~A}-\mathrm{C}$ ). The western blot results after stroke demonstrate that $\mathrm{NrF2} / \mathrm{HO}-1$ and $\mathrm{Bcl} 2$ significantly decline $(P<0.05$, Fig. $3 A)(P<0.001$, Fig. 3B) $(P<0.05$, Fig. 4 B), respectively, meanwhile, Bax and caspase 3 significantly increase $(P<0.001$, Fig $4 A)(P<0.01$, Fig. $4 C)$, respectively. On other hand, the TQ+stroke group shows a remarkable increase in NrF2/HO-1 and $\mathrm{Bcl} 2(\mathrm{P}<0.001)$ (Fig. $3 \mathrm{~B}, \mathrm{C}$, and Fig. 4B) as well as decreases in Bax and caspase3 level $(P<0.001)$ compared with the stroke group ( Fig. 4 A\& $C$ ). These outcomes are consistent with the immunofluorescence staining analysis of Nrf2/HO-1 as shown in Fig. $3 \mathrm{C}-\mathrm{F}$, which confirms the impact of TQ to attenuate both oxidative stress and cell death following brain injury [28].

\section{TQ reduce inflammation and excessive autophagy 7 days after reperfusion}


Autophagy and inflammation are collectively responded to stimulation by cerebral ischemic injury [29]. The obtained results illustrate demonstrate that TQ possess a great efficiency to reduce inflammation and attenuate excessive autophagy response after stroke. Fig 5C and Fig. 6A. represent the western blot of both autophagy and inflammatory factors. The obtained results illustrate that the pro-inflammatory factors iNOS, NF-kB are significantly decreased in TQ+stroke mice $(P<0.001)$ with a significant elevation in IL-10 expression $(P<0.001)$ compared with stroked mice (Fig.6B-D). The qPCR (Fig. 6F) results agree well with the western blot analysis. In brief, iNOS upregulates after stroke $(P<0.001)$ and decreases significantly in the TQ+Stroke group $(P<0.001)$. Autophagy proteins $(L C 3, B e c l i n-1, A T G 5)$ expression are significantly elevated after 7 days of stroke compared with the normal group $(P<0.01$, Fig.5D) $(P<0.01$, Fig. 5E) ( $P<0.001$, Fig. 5F), respectively. Also, TQ drug reduces the expression of LC3 $(P<0.001)$, Beclin-1 $(P<0.001)$, and ATG5 $(P<0.01)$ in TQ+Stroke mice. Meanwhile, both Immunofluorescence staining of the LC3 antibody (Fig. 5A-B) and qPCR (Fig. 5E) results of the ATG5 gene display a similar trend as the western blot results.

\section{The effect of TQ on the expression of proliferation and apoptosis-related proteins}

Next, we examine TrkB/AKT/mTOR signaling pathway proteins, c-myc protein-related apoptosis, Erk/pErk, MAP2, and PSD-95 to identify the proteins contribute to the progress of the brain ischemia. Our results illustrate that TQ have a large capability to reduce the cell death process and upregulate the expression of the proliferation proteins (Supplementary section 4 Fig. V and Fig. VI).

\section{TQ administration modulating astrocyte activity after 7 days from stroke}

The investigation of Immunofluorescence and western blot tests based on GFAP can be used to study the brain-specific astrocytic activity after stroke under the effect of TQ drug, which serves as an effective biomarker for acute stroke differential diagnosis (Fig.. 7A-F). As shown, the GFAP activity after stroke reveals a significant activation $(P<0.001)$, while inhibition is observed for the TQ+Stroke mice $(P<0.001)$ (Fig. 7A-E). The difference between the resting and activating state of microglia is displayed in Fig. $7 \mathrm{~B}$ and $\mathrm{C}$.

\section{Effect of TQ and TP on the relative proportion of YFP reportercells after stroke}

To determine the relative proportion of YFP reporter cells within the ischemic striatum, histological sections from YFP mice of all groups are utilized for co-labeling with NeuN immunofluorescence (Fig. 7 $\mathrm{G})$. The expression of mature neuronal marker (NeuN) of YFP cells within the injured striatum is rarely observed after 1 week from model compared to the normal group $(\mathrm{P}<0.05$, Fig. $7 \mathrm{H})$, but NeuN expression interestingly upregulates in the TQ+Stroke group $(P<0.001)$. This implies that TQ possesses a bifunctional therapeutic and protective effect on the ischemic brain.

\section{$T Q$ reduce the neuronal damaged area after stroke}

Fig. 8A-E presents the loss of neurons in the stroked area and dendrite spine obtained by a confocal microscope for YFP mice. As shown, the dendrite spines density decreases noticeably after the stroke 
model $(P<0.001)$ compared with the normal group, while TQ+Stroke mice display an obvious increase $(P<$ 0.01) compared with the stroke group. To deeply illustrate the most prominent spines, a reconstruction method of neurons implemented (Fig.8B). Observation indicates a marked decrease of mushroom and filopodia spines in stroked mice $(P<0.05)$ compared with normal mice, meanwhile, a remarkable increase of these spines in the TQ+Stroke group $(P<0.01)$ is noticed compared with the stroke group (Fig.8E).

\section{Pre-treatment by $T Q$ assisted cells number and shape recovery after OGD/R}

The difference in PC12 cell number and shape between the established groups can be seen in Supplementary Fig. IV. The spindle shape of PC12 cells and their interfacial connection after the OGD model are lost. The number of these cells decreases significantly $(P<0.001)$ after OGD compared with normal cells, while both TQ $(P<0.05)$ and TP $(P<0.01)$ attenuate this effect and recover the cells density and shape compared with OGD assisted PC12 cells (Fig. VII (B)).

\section{Impact of pre-treatment by TQ on cells death rate following OGD/R}

The effect of both TQ and TP drugs on PC12 after the OGD model is addressed by Tunnel assay and flow cytometry as provided in Fig. 9. The apoptosis analysis for cell death rate after TQ or TP addition ( $24 \mathrm{~h}$ prior of OGD model), show a significant reduction $(P<0.01)(P<0.001)$, respectively (Fig. 9 A-E). Hence, TQ treatment $24 \mathrm{~h}$ before OGD has a great potential to relieve cell death in vitro.

\section{TQ alleviates OGD/R induced inflammation via activation of the antioxidant $\mathrm{Nrf2/HO}-1$ pathway and inhibition of NF-kB.}

Immunofluorescence staining of both $\mathrm{Nrf} / \mathrm{HO}-1$ and $\mathrm{NfkB}$ expressions in vitro indicate that $\mathrm{TQ}$ can alleviate the OGD/R induced inflammation via Nrf2/HO-1 pathway Immunofluorescence staining of both $\mathrm{Nrf2/HO}-1$ expressions in vitro shows a significant reduction in their levels after the OGD model $(P<0.05)$ $(P<0.01)$, respectively compared with normal cells (Fig.10A-D). Whilst TQ treated cells $24 \mathrm{~h}$ before OGD exhibit a significant upregulation in Nrf2/HO-1 expression $(\mathrm{P}<0.001)$ compared with OGD assisted PC12 cells as shown in Fig.10A-D. Additionally, immunofluorescence staining of proinflammatory marker NF-kB displays a significant alleviation of NF-kB positive cells in TQ+OGD cells $(P<0.001$, Fig.10 E- F) compared with OGD assisted PC12 cells, and thus demonstrate that TQ can attenuate inflammation through Nrf2/HO-1 pathway (Fig.10A-F).

\section{Discussion}

The long-term adult palsy can be dramatically triggered by a stroke. It is well known that inflammation, autophagy, and apoptosis processes have a robust effect on stoke disease [30]. Recently, the treatment of ischemic stroke has been mainly accomplished via neuroprotection and reperfusion, however many obstacles are still existed due to variation in stroke severity and instability as well as limitation of using some drugs [31]. Thus, there is an urgent need for more applicable treatment options. Based on these findings, we speculated that TQ therapy can be employed as an alternative candidate to effectively treat 
brain stroke. The harvested results signify that TQ can attenuate the motor deficit after stroke, reduce inflammation, and neuronal cell death induced by the photo-thrombosis model in mice. The neuroprotective effects of TQ are strongly correlated with the activation of Nrf2/HO-1 and TrkB/AKT/mTOR and these expressions contribute to modulate the inflammatory and autophagy responses of the nervous system. Recent studies demonstrated that drugs with complex mechanisms of action, which promote neuro-recovery are a better choice to treat acute ischemic stroke than neuroprotective drugs with a single mechanism of action [32]. Over the past decades, TP has been adopted as an ischemic therapy. However, the wide-spread use of TP is still limited, which has postponed the clinical development and trials [33]. Side-by side, several studies reported the efficiency of TQ as an anticancer, anti-inflammatory, and antioxidant drug due to its bioactive properties [34]. The present study offers new chances toward the investigation of TQ as neuromodulator therapy in a mouse model of focal cerebral ischemia to replace the conventional TP drug. To achieve our aim, we initially ascertain the toxicity effect of TQ on liver tissue compared with TP drug as indicated by generated fibrosis. This observation agrees with those reported in the literature, which evidenced the hepatoprotective role of TQ and liver toxicity of TP [35]. Additionally, our behavioral tests of mice injected by TQ 5 days before the stroke model exhibit a significant improvement in their motor dysfunction resulting from the ischemic model, raising in survival rate without changes in body weight. Moreover, histological analyses report that TQ therapy can sufficiently decrease the infraction area and recover the brain tissue morphology after stroke as well as increase the neural cell number within the infarct area [36]. Reported data disclosed the vital role of increasing the Nrf2 protein which associates with the antioxidant responsive element (ARE) and can attenuate the oxidative stress damage through releasing HO-1 into the cytoplasm. This points out the ability of NrF2/HO-1 to suppress oxidative stress following stroke [37].

In vivo and in vitro results show obvious mitigation of brain damage with TQ treatment after stroke through activating the Nrf2/HO-1 pathway, which in turn increases the HO-1 within the cytoplasm and consequently modulates both of apoptosis, autophagy, and inflammatory processes. Furthermore, analysis based on in vivo and in vitro data of the TP+Stroke group displays an insignificant change in HO-1 protein expression, while Nrf2 expression of the TQ+Stroke group exhibits a higher level than the $\mathrm{TP}+$ Stroke group because of the non-ability of TP drug to activate the Nrf2/HO-1 pathway [38]. It has been proven that TQ possesses a unique anti-inflammatory activity [39]. Along with this, the in vivo and in vitro usage of TQ before establishing the model shows a significant downregulation in inflammatory mediators such as cytokines and chemokines (i.e. NF-kB/iNOS and PSD-95). The increase of these mediators releases nitric oxide (NO) substance and thereby accelerates ischemic brain damage. In sharp contrast, the TQ+Stroke group reveals an increment of IL-10 anti-inflammatory protein, which fits well with the previous reports that indicated the effect of stroke in inducing the anti-inflammatory process [40]. Previous studies suggested that autophagy-targeted therapy plays a role as a promising therapeutic approach for neurodegenerative diseases, especially stroke [41]. Our analysis shows an intensive autophagy marker (LC3, Beclin, and ATG) levels for both stroked mice and TP+Stroke mice. Unlike, TQ+Stroke mice display lower levels of autophagy markers, consistent with previously reported data, which showed that autophagy served as a double-edged sword. It has been established that the natural 
neuroprotective agent possesses a protective property against ischemic stroke through inhibiting autophagy by impeding the expression of Beclin-1 and activating the PI3K/Akt pro-survival pathway [42]. Ischemia-reperfusion injury induces neuronal cell death within the injured area throughout increasing apoptotic related proteins Bax and this triggers caspase 3 to be released from mitochondria by inhibiting $\mathrm{Bcl} 2$. Also, the upregulation of early apoptotic genes c-fos and c-myc proteins can enhance caspase3 [43].

Our in vivo results confirm that both of Bax/Caspase3/c-FOS and c-MYC proteins are highly expressed after stroke, but TQ+Stroke and TP+Stroke mice show an interesting downregulation of these proteins associated with an upregulation in $\mathrm{BCl}-2$ expression. Similarly, a significant reduction in cell death within TQ or TP-treated cells $24 \mathrm{~h}$ before the OGD model [44]. The TrkB/AKT/mTOR proteins have been addressed as a neuron pro-survival pathway [45]. The collected data indicate a significant upregulation of the TrkB/AKT/mTOR proteins pathway in the TQ+Stroke group compared with TP+Stroke and Stroke groups, consistent with the introduced hypothesis [46].

Moreover, 5-HT gene has aroused increasing interest in the stroke pathophysiology research field and affects the neurotransmitter system of the brain. Increased activity of 5-HT in the brain after stroke is lifethreatening [47]. Our q-PCR results demonstrate that the expression of the 5-HT gene is significantly upregulated after stroke while a lower level of this gene expression can be attained for TQ+Stroke mice, implying that TQ has a good ability to protect the brain from stroke pathophysiological progress. Besides, TQ therapy displays a large efficiency to modulate the activity of the astrocytes after stroke. This notification constitutes with that reported previously [48].

Analysis indicates that TQ increases the expression of NeuN within YFP reporter cells in the ischemic hemisphere and recovers dendritic spines density, suggesting its importance in promoting neurogenesis in damaged neuron area. It is worth mentioning that brain ischemia can induce changes in dendritic branch complexity and spine density [49]. Our analyzed data elucidate that the dendritic spines density significantly reduced after stroke and this effect is reversible in TQ and TP mice. Additionally, both mushroom and filopodia spines possess a higher population number in TQ+Stroke mice, while a lower number in stroked and TP+ Stroke mice is evident. The abundance of these types may refer to developing neurons process [50]. Previous studies claimed mushroom spines act like memory spines [51] These spines are known to have larger postsynaptic densities and were proposed to recruit more a-Amino-3hydroxy-5-methyl-4-isoxazole propionic acid (AMPA) receptors [52]. The neuromodulator effects of TQ (Supplementary Fig. VIII) and their roles in amelioration of stroke outcome represent a substantial mechanism and thereby this drug may provide the neuroprotection in acute neurodegenerative conditions. In this context, the efficacy of a prolonged dose of TQ evidences its activity compared with TP drugs.

\section{Conclusion}


The analyzed results confirmed the efficiency of TQ drug as a protective therapy against cerebral brain ischemia without any detectable toxic effect under a chronic dose by increasing proliferating protein expression, decreasing inflammation and neuronal cell death as well as controlling the autophagy process (Supplementary Fig. IX). Although more fundamental studies are required to confirm the effect of different doses of TQ on stroke and its lethal dose. Moreover, systematic in vitro investigations can be afforded to deeply explore the potent dose of TQ drug, and its effect on different cell lines.

\section{Abbreviations}

ROS; Reactive Oxygen Species; TQ: Thymmoquninone; TP: Triptolide; mTOR: mammalian target of rapamycin: Erk1/2; extracellular signal-regulated kinases; p-Erk1/2: phospho-Erk1/2; Nrf2:nuclear factor erythroid 2-related factor 2; HO-1: Heme oxygenase 1; GFAP: Glial fibrillary acidic protein; PC12: cell line derived from a pheochromocytoma of the rat adrenal medulla; OGD/R: oxygen glucose deprivation/reoxygenation; NF-kB: Nuclear Factor kappa-light-chain-enhancer of activated B cells; iNOS: Inducible nitric oxide synthase; Bax: Apoptosis regulator BAX, also known as bcl-2-like protein 4; Bcl2: Bcell lymphoma 2; C-FOS: proto-oncogene C-Myc: proto-oncogene ;PSD-95: postsynaptic density protein; 95MAP2: Microtubule Associated Protein 2; IL-10: Interleukin 10; TrkB :Tropomyosin receptor kinase B; AKT: Protein kinase B (PKB) serine/threonine-specific protein kinase; IRAK1: Interleukin 1 Receptor Associated Kinase 1; LC3: Microtubule-associated protein 1A/1B-light chain 3; Atg5: Autophagy related 5; Beclin-1: mammalian orthologue of yeast Atg6; 5-HT: 5-hydroxytryptamine receptors; thy1-YFP: transgenic mice express yellow fluorescent protein at high levels in motor and sensory neurons;C57BL/6J: inbred strain of laboratory mouse; DMSO: Dimethyl sulfoxide ; PBS: Phosphatebuffered saline; NeuN: Neuronal nuclei protein (Fox-3, Rbfox3, or Hexaribonucleotide Binding Protein-3); RIPA: Radioimmunoprecipitation assay buffer; BCA: Bicinchoninic acid assay; PVDF: polyvinylidene difluoride.

\section{Declarations}

\section{Acknowledgements}

Not applicable

\section{Authors' Contributions}

Marong Fang and Zhiying Hu designed the experiments. Nashwa Amin performed the experiment, drafted the manuscript, and analyzed the data. Qiannan Ren participated in the in vitro study. Xiaoxue Du, Shijia Chen, Benson O.A. Botchway, and Azhar B. Hussien are mainly responsible for polishing the article and manuscript editing and writing. Marong Fang is mainly responsible for obtained funding. All authors read and approved the final manuscript.

\section{Sources of funding}


The National Natural Science Foundation of China (grant numbers 81671138 and 81871063 ) supported this study. We extend our appreciation to the Core Facilities of Zhejiang University Institute of Neuroscience for their technical assistance, facilities, and support throughout this study.

\section{Availability of data and materials}

The datasets during and/or analyzed during the current study are available from the corresponding author on reasonable request.

\section{Ethics approval and consent to participate}

This study was performed following the Guide of Care and Use of Laboratory Animal Center of Zhejiang University, China. All animal treatments were approved by the Ethics Committee for Use of Experimental Animals at Zhejiang University.

\section{Consent for publication}

Not applicable.

\section{Competing interests}

The authors declare that they have no known competing financial interests or personal relationships that could have appeared to influence the work reported in this paper

\section{References}

1. Moskowitz MA, Lo EH, ladecola C (2010) The science of stroke: mechanisms in search of treatments. Neuron. 67:181-198.

2. Stefanidou M, Das RR, Beiser AS, Sundar B, Kelly-Hayes M, Kase CS, Devinsky O, Seshadri S, Friedman D (2017) Incidence of seizures following initial ischemic stroke in a community-based cohort: The Framingham Heart Study. Seizure 47:105-110.

3. Barone, F.C (2010) Post-stroke pharmacological intervention: Promoting brain recovery from injury in the future. Neuropharmacology 1-3.

4. Benz CC, Yau C (2008) Ageing, oxidative stress, and cancer: paradigms in parallax. Nat Rev Cancer 8 :875-879.

5. Rojo A, McBean G, Cindric M, Egea J, López MG, Rada P, Zarkovic N, Cuadrado A (2014) Redox control of microglial function: molecular mechanisms and functional significance. Antioxid. Redox Signal 21: 1766-1801. doi: 1089/ars.2013.5745.

6. Wen HL, Liang ZS, Zhang R, Yang K (2013) Anti-inflammatory effects of triptolide improve left ventricular function in a rat model of diabetic cardiomyopathy. Cardiovasc Diabetol 12:50.

7. Yao J, et al (2008) Involvement of mitochondrial pathway in triptolide-induced cytotoxicity in human normal liver L-02 cells. Biol Pharm Bull 31 (4) :92-597. 
8. Sun L, et al (2013) Triptolide alters barrier function in renal proximal tubular cells in rats. Toxicol Lett 223 (1):96-102.

9. Shao-xi W, Ning-ru G (2005) Clinical observation on effect of triptolide tablet in treating patients with psoriasis vulgaris. Chinese Journal of Integrative Medicine 11(2):147-8.

10. Kanter M, Coskun O, Uysal H. (2006). The antioxidative and antihistaminic effect of Nigella sativa and its major constituent, thymoquinone on ethanol-induced gastric mucosal damage. Archives of toxicology, 80(4): 217-224.

11. Fouda, A. M., Daba, M. H., Dahab, G. M., \& Sharaf El-Din, O. A. (2008). Thymoquinone ameliorates renal oxidative damage and proliferative response induced by mercuric chloride in rats. Basic \& clinical pharmacology \& toxicology, 103(2), 109-118.

12. Hossen, M. J., Yang, W. S., Kim, D., Aravinthan, A., Kim, J. H., \& Cho, J. Y. (2017). Thymoquinone: An IRAK1 inhibitor with in vivo and in vitro anti-inflammatory activities. Scientific reports 7: 42995.

13. Alhebshi, A. H., Odawara, A., Gotoh, M., \& Suzuki, I. (2014). Thymoquinone protects cultured hippocampal and human induced pluripotent stem cells-derived neurons against a-synuclein-induced synapse damage. Neuroscience letters, 570, 126-131.

14. Jin XQ, Ye F, Zhang JJ, Zhao Y, Zhou XL. Triptolide attenuates cerebral ischemia and reperfusion injury in rats through the inhibition the nuclear factor kappa B signaling pathway. Neuropsychiatr Dis Treat. 2015; 11:1395-1403. Published 2015 Jun 3. doi:10.2147/NDT.S82052

15. Zhu N, Ruan J, Yang X, Huang,Y, Jiang Y, Wang Y, Cai D, Geng Y, Fang M (2019) Triptolide improves spinal cord injury by promoting autophagy and inhibiting apoptosis. Cell Biol Int 44:785-794.

16. Bai S, Hu Z, Yang Y, Yin Y, Li W, Wu L, Fang M (2015) Anti-Inflammatory and Neuroprotective Effects of Triptolide via the NF-KB Signaling Pathway in a Rat MCAO Model. The Anatomical Record 299(2) :256-266.

17. Watson, B. D., Dietrich, W. D., Busto, R., Wachtel, M. S., \& Ginsberg, M. D. (1985). Induction of reproducible brain infarction by photochemically initiated thrombosis. Annals of neurology, 17(5), 497-504.

18. Watson, B. D., Dietrich, W. D., Busto, R., Wachtel, M. S., \& Ginsberg, M. D. (1985). Induction of reproducible brain infarction by photochemically initiated thrombosis. Annals of neurology, 17(5), 497-504.

19. Macrae, I. M., Robinson, M. J., Graham, D. I., Reid, J. L., \& McCulloch, J. (1993). Endothelin-1-induced reductions in cerebral blood flow: dose dependency, time course, and neuropathological consequences. Journal of cerebral blood flow and metabolism: official journal of the International Society of Cerebral Blood Flow and Metabolism, 13(2), 276-284.

20. Park SY, Marasini S, Kim GH, et al. A method for generating a mouse model of stroke: evaluation of parameters for blood flow, behavior, and survival Exp Neurobiol. 2014;23(1):104-114.

21. Li, X., Blizzard, K. K., Zeng, Z., DeVries, A. C., Hurn, P. D., \& McCullough, L. D. (2004). Chronic behavioral testing after focal ischemia in the mouse: functional recovery and the effects of gender. Experimental neurology, 187(1), 94-104. 
22. Barth AM, Mody I. Novel test of motor and other dysfunctions in mouse neurological disease models. J Neurosci Methods. 2014; 221:151-158.

23. Claudia Gomez, Jacinto Santiago-Mejia, Rosa Ventura-Martinez, and Rodolfo Rodriguez. The Sunflower Seed Test: A Simple Procedure to Evaluate Forelimb Motor Dysfunction After Brain Ischemia, DRUG DEVELOPMENT RESEARCH 67:752-756 (2006).

24. Amin N, Xie S, Tan X, Chen Y, Ren Q, Benson OAB, Hu S, Y Ma Y, Hu Z, Fang M (2020) Optimized integration of fluoxetine and 7, 8-dihydroxyflavone as an efficient therapy for reversing depressivelike behavior in mice during the perimenopausal period. Progress in Neuropsychopharmacology \& Biological Psychiatry 101: 109939.

25. Back T, Hemmen T, Schuler OG. Lesion evolution in cerebral ischemia. J Neurol. 2004; 251:388-397.

26. Rena Q, Hu Z, Jiang Y, Tan X, Benson O A B, Amin N, Lin G, Yu G , Fang M (2019) SIRT1 Protects Against Apoptosis by Promoting Autophagy in the Oxygen Glucose Deprivation/Reperfusion-Induced Injury. Front. Neurol. 10:1289.

27. Back T, Hemmen T, Schuler OG. Lesion evolution in cerebral ischemia. J Neurol. 2004; 251:388-397.

28. Alhebshi AH, Gotoh M, Suzuki I (2013) Thymoquinone protects cultured rat primary neurons against amyloid induced neurotoxicity, Biochem. Biophys. Res.Commun. 433: 362-367.

29. Babazadeh B, Sadeghnia HR, Safarpour Kapurchal E, Parsaee H, Nasri S, Tayarani-Najaran Z. Protective effect of Nigella sativa and thymoquinone on serum/glucose deprivation-induced DNA damage in PC12 cells. Avicenna J Phytomed. 2012;2(3):125-132. Schaller and R. Graf, "Cerebral ischemia and reperfusion: the pathophysiologic concept as a basis for clinical therapy," Journal of Cerebral Blood Flow \&Metabolism, vol. 24, no. 4, pp. 351-371, 2004.

30. Sun L, Kuroiwa T, Ishibashi S, Miki K, Li S, Xu H, Endo S, Mizusawa H. Two region-dependent pathways of eosinophilic neuronal death after transient cerebral ischemia. Neuropathology. 2009 Feb; 29(1):45-54.

31. Cyrus Jalili, Maryam Sohrabi , Faramarz Jalili , Mohammad Reza Salahshoor . Assessment of thymoquinone effects on apoptotic and oxidative damage induced by morphine in mice heart Cell Mol Biol (Noisy-le-grand). 2018 Jun 30;64(9):33-38.

32. Zhou X, Zhou J, Li X, Guo C, Fang T, Chen Z. GSK-3 $\beta$ inhibitors suppressed neuroinflammation in rat cortex by activating autophagy in ischemic brain injury. Biochem Biophys Res Commun. $2011 \mathrm{Jul}$ 29;411(2):271-5. doi: 10.1016/j.bbrc.2011.06.117. Epub 2011 Jun 23. PMID: 21723251.

33. Chen Y, Meng J, Xu Q, Long T, Bi F, Chang C, Liu W. Rapamycin improves the neuroprotection effect of inhibition of NLRP3 inflammasome activation after TBI. Brain Res. 2019; 1710:163-172.

34. Schaller and R. Graf, "Cerebral ischemia and reperfusion: the pathophysiologic concept as a basis for clinical therapy," Journal of Cerebral Blood Flow \&Metabolism, vol. 24, no. 4, pp. 351-371, 2004.

35. Dafin F. Muresanu1,2 - Stefan Strilciuc1,2 - Adina Stan Current Drug Treatment of Acute Ischemic Stroke: Challenges and Opportunities. CNS Drugs (2019) 33:841-847.

36. Zhenyan Hou1,2, Lei Chen1,2, Pingfei Fang1,2, Hualin Cai1,2, Huaibo Tang3, Yongbo Peng4, Yang Deng5, Lingjuan Cao1,2, Huande Li1,2, Bikui Zhang1,2* and Miao Yan . Mechanisms of Triptolide- 
Induced Hepatotoxicity and Protective Effect of Combined Use of Isoliquiritigenin: Possible Roles of Nrf2 and Hepatic Transporters. Front. Pharmacol., 16 March 2018, 9:226.

37. Galaly S.R., Ahmed O.M., Mahmoud A.M., Thymoquinone and curcumin prevent gentamicin-induced liver injury by attenuating oxidative stress, inflammation and apoptosis, J. Physiol Pharmacol., 2014, $65,823-832$.

38. Woo CC, Hsu A, Kumar AP, Sethi G, Tan KH. Thymoquinone inhibits tumor growth and induces apoptosis in a breast cancer xenograft mouse model: the role of p38 MAPK and ROS. PLoS One. 2013;8(10): e75356. Published 2013 Oct 2. doi: 10.1371/journal.pone.0075356.

39. Alhebshi AH, Gotoh M, Suzuki I. Thymoquinone protects cultured rat primary neurons against amyloid $\beta$-induced neurotoxicity. Biochem Biophys Res Commun (2013) 433(4):362-7.

40. Kanter M. Nigella sativa and derived thymoquinone prevents hippocampal neurodegeneration after chronic toluene exposure in rats. Neurochem Res. 2008;33(3):579.

41. Flamment $M$, Hajduch E, Ferre $P$, et al. New insights into ER stress-induced insulin resistance. TrendsEndocrinol Metab. 2012; 23:381-390.

42. Huuskonen MT, Loppi $\mathrm{S}$, Dhungana $\mathrm{H}$, et al.Bexarotene targets autophagy and is protective against thromboembolic stroke in aged mice with tauopathy. Sci Rep. 2016; 6:33176.

43. Lu A, Tang Y, Ran R, Clark JF, Aronow BJ, Sharp FR (2003) Genomics of the periinfarction cortex after focal cerebral ischemia. J Cereb Blood Flow Metab 23:786-810.

44. Ullah I, Ullah N, Naseer MI, Lee HY, Kim MOK. Neuroprotection with metformin and thymoquinone against ethanol-induced apoptotic neurodegeneration in prenatal rat cortical neurons. BMC Neurosci (2012) 13:11.

45. Ai Chen, Li-Jing Xiong, Yu Tong, Meng Mao. Neuroprotective effect of brain-derived neurotrophic factor-mediated by autophagy through the PI3K/Akt/mTOR pathway. MOLECULAR MEDICINE REPORTS 8: 1011-1016, 2013.

46. Pengzhou Hanga, Jing Zhaoc, Li Sunc, Minghui Li, Yu Han, Zhimin Dua, Yue Lib, Brain-derived neurotrophic factor attenuates doxorubicin-induced cardiac dysfunction through activating Akt signaling in rats. J. Cell. Mol. Med. Vol 21, No 4, 2017 pp. 685-696.

47. Bode-Greuel KM, Klisch J, Glaser T, Traber J: Serotonin 5-HT, Areceptor agonists as neuroprotective agents in cerebral ischemia, inKrieglstein $\mathrm{J}$, Oberpichler $\mathrm{H}$ (eds): Pharmacology of Cerebral Isch-emia. Stuttgart, Wissenschaftliche Verlagsgesellsch, 1990, pp 485-491.

48. Bourne JN, Harris KM. Coordination of size and number of excitatory and inhibitory synapses results in a balanced structural plasticity along mature hippocampal CA1 dendrites during LTP. Hippocampus. 2011;21(4):354-373. doi:10.1002/hipo.20768.

49. Fiala JC, Feinberg M, Popov V, Harris KM (1998) Synaptogenesis via dendritic filopodia in developing hippocampal area CA1. J Neurosci 18: 8900-8911.

50. Tashiro A, Yuste R (2003) Structure and molecular organization of dendritic spines. Histol Histopathol 18: 617-634. 
51. Matsuzaki M, Ellis-Davies GC, Nemoto T, Miyashita Y, lino M, Kasai H. Dendritic spine geometry is critical for AMPA receptor expression in hippocampal CA1 pyramidal neurons. Nat Neurosci. 2001;4(11):1086-1092. doi:10.1038/nn736.

\section{Figures}
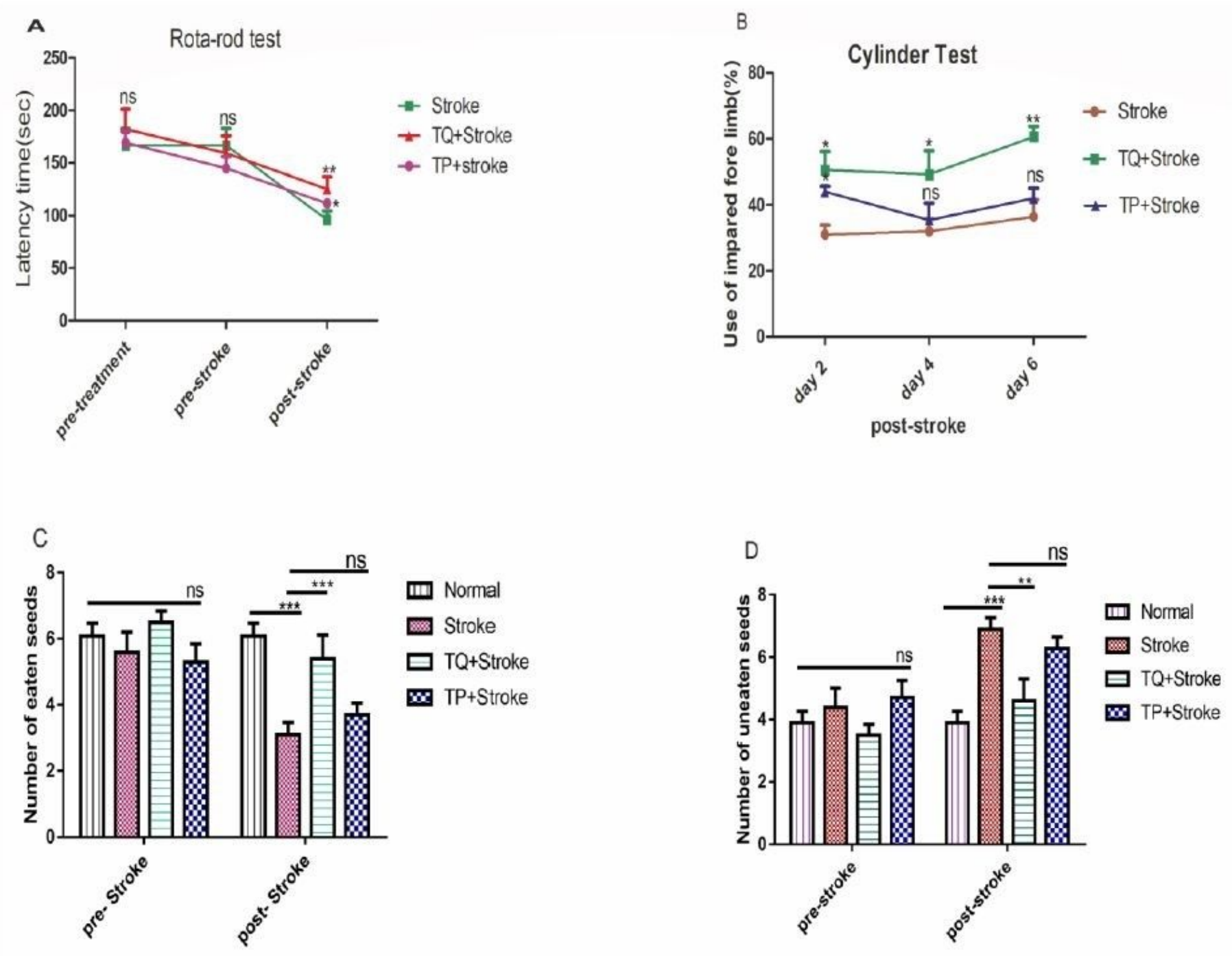

Figure 1

TQ therapy attenuates motor dysfunction after stroke. (A) Rotarod test; Latency time, TQ+ Stroke Vs. Stroke ${ }^{*} \mathrm{P}<0.01, T P+$ Stroke Vs. Stroke $" P<0.05$. (B) Cylinder test; Percentage of the impaired forelimb, day 2; TQ+ Stroke Vs. Stroke * $P<0.05, T P+$ Stroke Vs. Stroke *P<0.05, day 4; TQ+Stroke Vs. Stroke *P<0.05, TP+ Stroke Vs. Stroke $P=n s$, day 6; TQ+ Stroke Vs. Stroke "P<0.01, TP+ Stroke Vs. Stroke $P=n s$. (C) Sunflower test; Number of eaten seeds, (D)Number of uneaten seeds Stroke Vs. Normal "*P<0.001, $\mathrm{TQ}+$ Stroke Vs. Normal * $\mathrm{P}<0.01, \mathrm{TQ}+$ Stroke Vs. Normal $\mathrm{P}=\mathrm{ns}$. All values are expressed as mear $+\mathrm{SEM}$, Each group $n=10$ in each test. 

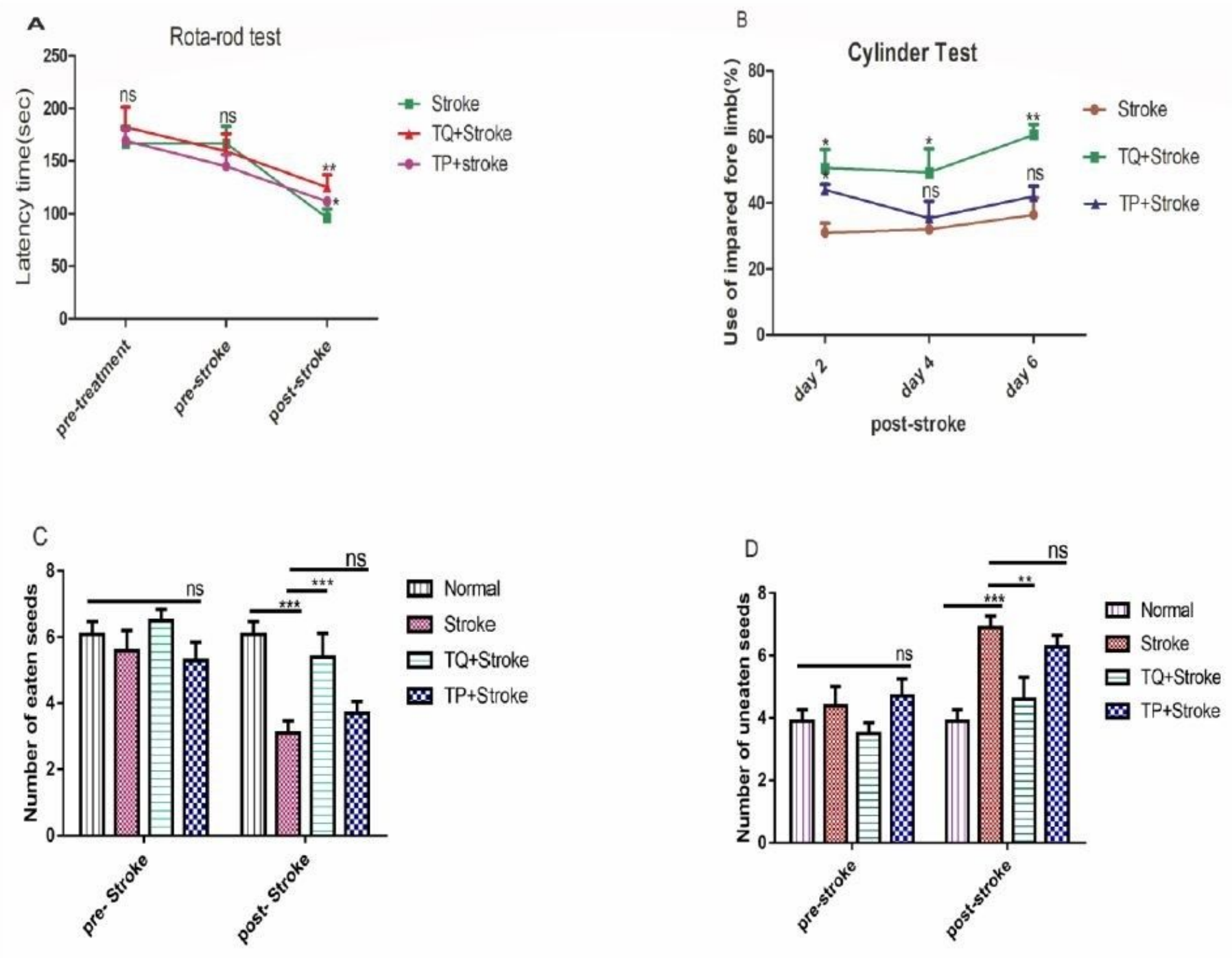

Figure 1

TQ therapy attenuates motor dysfunction after stroke. (A) Rotarod test; Latency time, TQ+ Stroke Vs. Stroke ${ }^{*} P<0.01, T P+$ Stroke Vs. Stroke " $P<0.05$. (B) Cylinder test; Percentage of the impaired forelimb, day 2; TQ+ Stroke Vs. Stroke * $P<0.05$, TP+Stroke Vs. Stroke *P<0.05, day 4; TQ+ Stroke Vs. Stroke ${ }^{*} \mathrm{P}<0.05, \mathrm{TP}+$ Stroke Vs. Stroke $\mathrm{P}=\mathrm{ns}$, day 6; TQ+ Stroke Vs. Stroke "P<0.01, TP+ Stroke Vs. Stroke $\mathrm{P}=$ ns. (C) Sunflower test; Number of eaten seeds, (D)Number of uneaten seeds Stroke Vs. Normal "* $P<0.001$, $\mathrm{TQ}+$ Stroke Vs. Normal *“ $\mathrm{P}<0.01, \mathrm{TQ}+$ Stroke Vs. Normal $\mathrm{P}=\mathrm{ns}$. All values are expressed as mear $+\mathrm{SEM}$, Each group $n=10$ in each test. 

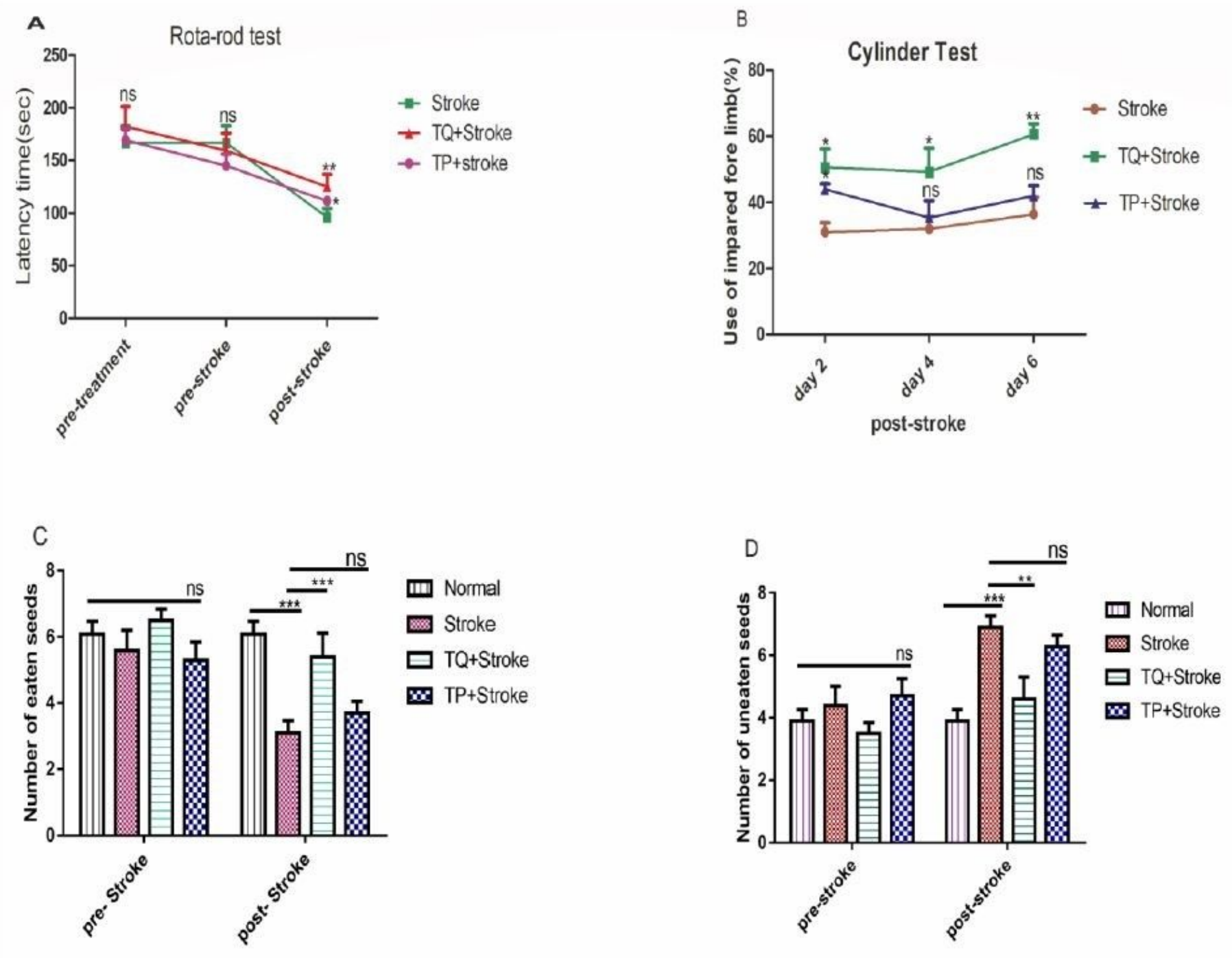

Figure 1

TQ therapy attenuates motor dysfunction after stroke. (A) Rotarod test; Latency time, TQ+ Stroke Vs. Stroke ${ }^{*} P<0.01, T P+$ Stroke Vs. Stroke " $P<0.05$. (B) Cylinder test; Percentage of the impaired forelimb, day 2; TQ+ Stroke Vs. Stroke * $P<0.05$, TP+Stroke Vs. Stroke *P<0.05, day 4; TQ+ Stroke Vs. Stroke ${ }^{*} \mathrm{P}<0.05, \mathrm{TP}+$ Stroke Vs. Stroke $\mathrm{P}=\mathrm{ns}$, day 6; TQ+ Stroke Vs. Stroke "P<0.01, TP+ Stroke Vs. Stroke $\mathrm{P}=$ ns. (C) Sunflower test; Number of eaten seeds, (D)Number of uneaten seeds Stroke Vs. Normal "* $P<0.001$, $\mathrm{TQ}+$ Stroke Vs. Normal *“ $\mathrm{P}<0.01, \mathrm{TQ}+\mathrm{Stroke}$ Vs. Normal $\mathrm{P}=\mathrm{ns}$. All values are expressed as mear + SEM, Each group $n=10$ in each test. 

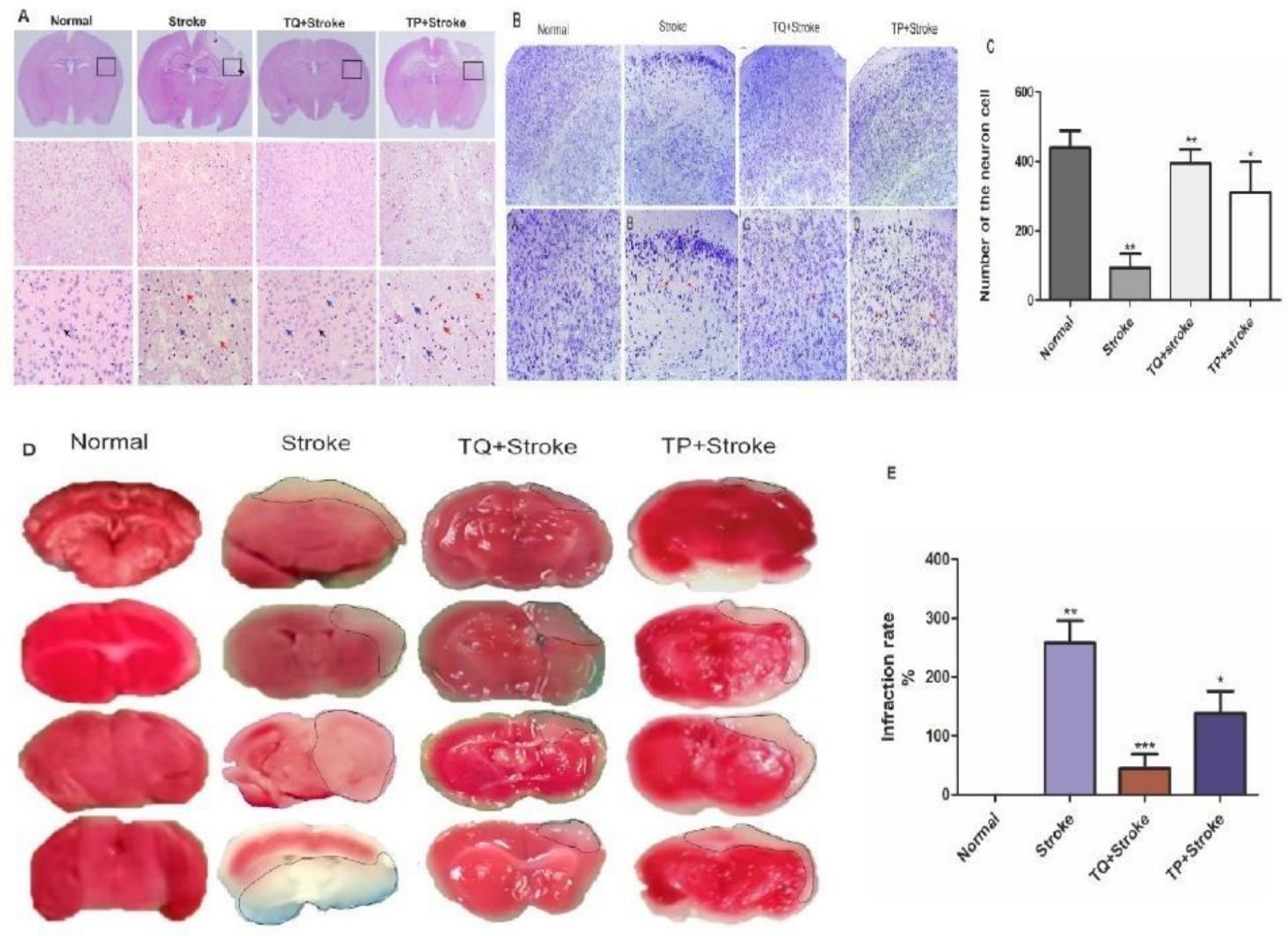

E

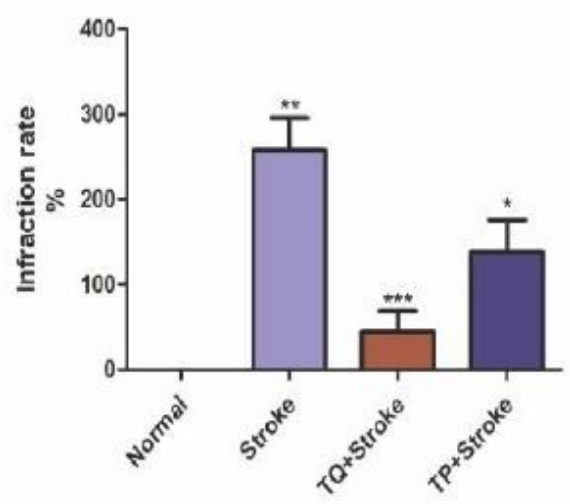

Figure 2

TQ therapy significantly reduce the infraction area and brain damage resulting from ischemic stroke. A, H\&E staining sections. The injured area is marked by a black square. Neuronal degeneration (blue arrow), vacuolated adjacent tissue (red arrow). B, represent Nissl staining sections. C, The number of neuron cells, Stroke Vs. Normal: ${ }^{*} P<0.01, T Q+$ stroke Vs. Stroke: "P<0.01, TP+Stroke Vs. Stroke: ${ }^{*} P<0.05$. D, TTC staining. E, Infraction area rate, Stroke Vs. Normal: "P<0.01, TQ+stroke Vs. Stroke: "*P<0.001, TP+ Stroke Vs. Stroke: ${ }^{*}<<0.05$. All values are expressed as mean $+S E M$, Each group $n=4$. 

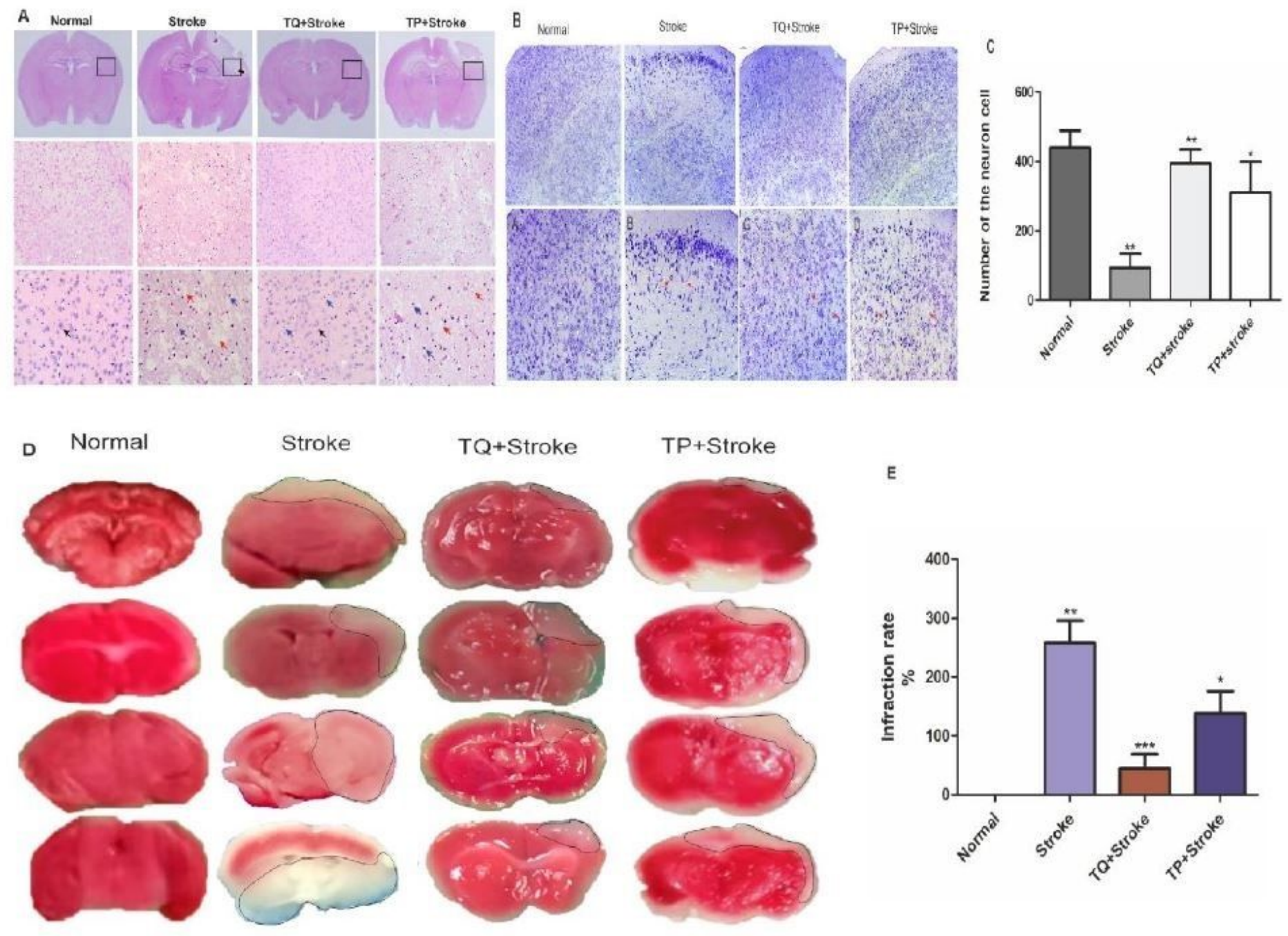

E

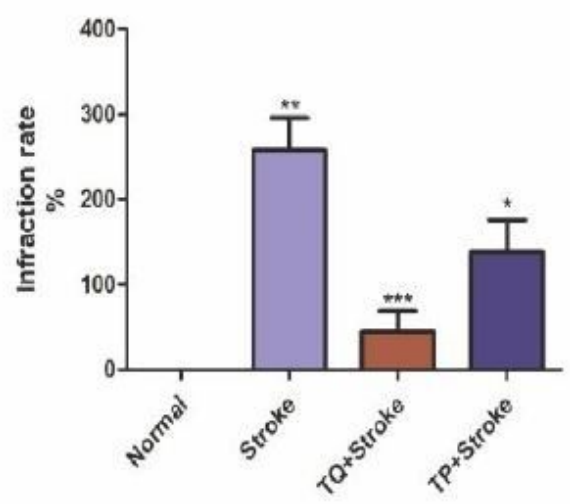

Figure 2

TQ therapy significantly reduce the infraction area and brain damage resulting from ischemic stroke. A, H\&E staining sections. The injured area is marked by a black square. Neuronal degeneration (blue arrow), vacuolated adjacent tissue (red arrow). B, represent Nissl staining sections. C, The number of neuron cells, Stroke Vs. Normal: ${ }^{*} P<0.01, T Q+$ stroke Vs. Stroke: "P<0.01, TP+Stroke Vs. Stroke: ${ }^{*} P<0.05$. D, TTC staining. E, Infraction area rate, Stroke Vs. Normal: "P<0.01, TQ+stroke Vs. Stroke: "*P<0.001, TP+ Stroke Vs. Stroke: ${ }^{*}<<0.05$. All values are expressed as mean $+S E M$, Each group $n=4$. 

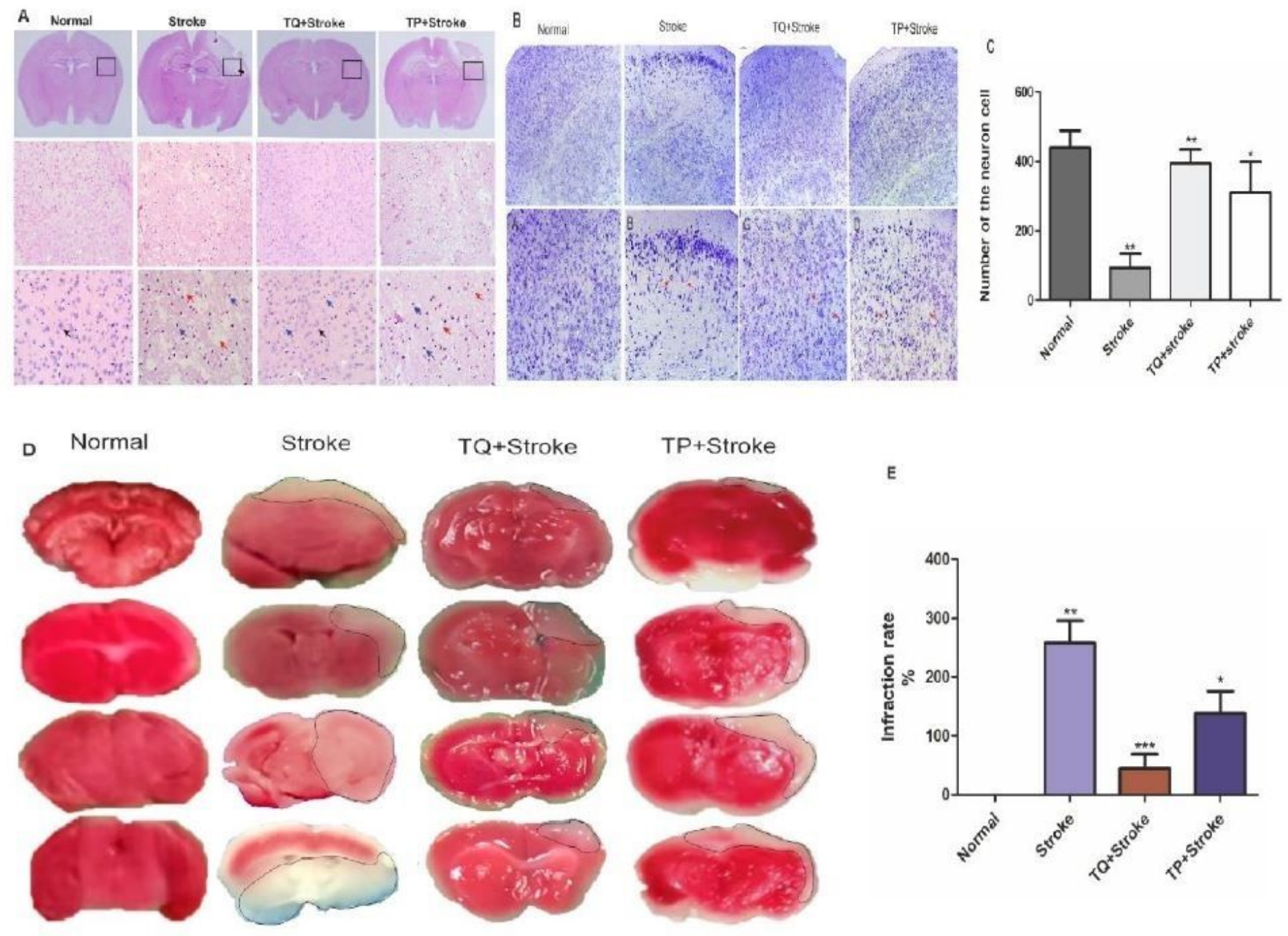

E

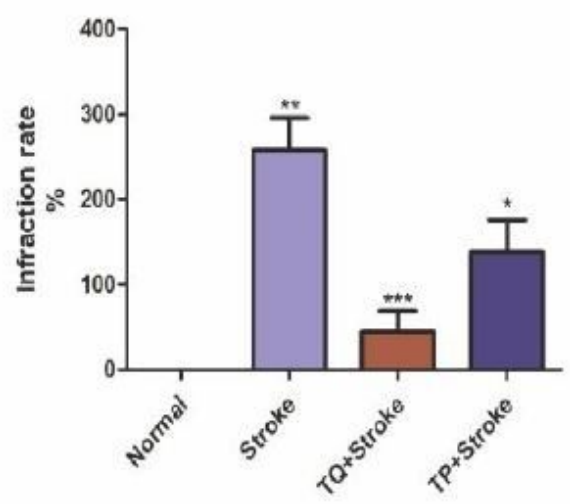

Figure 2

TQ therapy significantly reduce the infraction area and brain damage resulting from ischemic stroke. A, H\&E staining sections. The injured area is marked by a black square. Neuronal degeneration (blue arrow), vacuolated adjacent tissue (red arrow). B, represent Nissl staining sections. C, The number of neuron cells, Stroke Vs. Normal: ${ }^{*} P<0.01, T Q+$ stroke Vs. Stroke: "P<0.01, TP+Stroke Vs. Stroke: ${ }^{*} P<0.05$. D, TTC staining. E, Infraction area rate, Stroke Vs. Normal: "P<0.01, TQ+stroke Vs. Stroke: "*P<0.001, TP+ Stroke Vs. Stroke: ${ }^{*}<<0.05$. All values are expressed as mean $+S E M$, Each group $n=4$. 

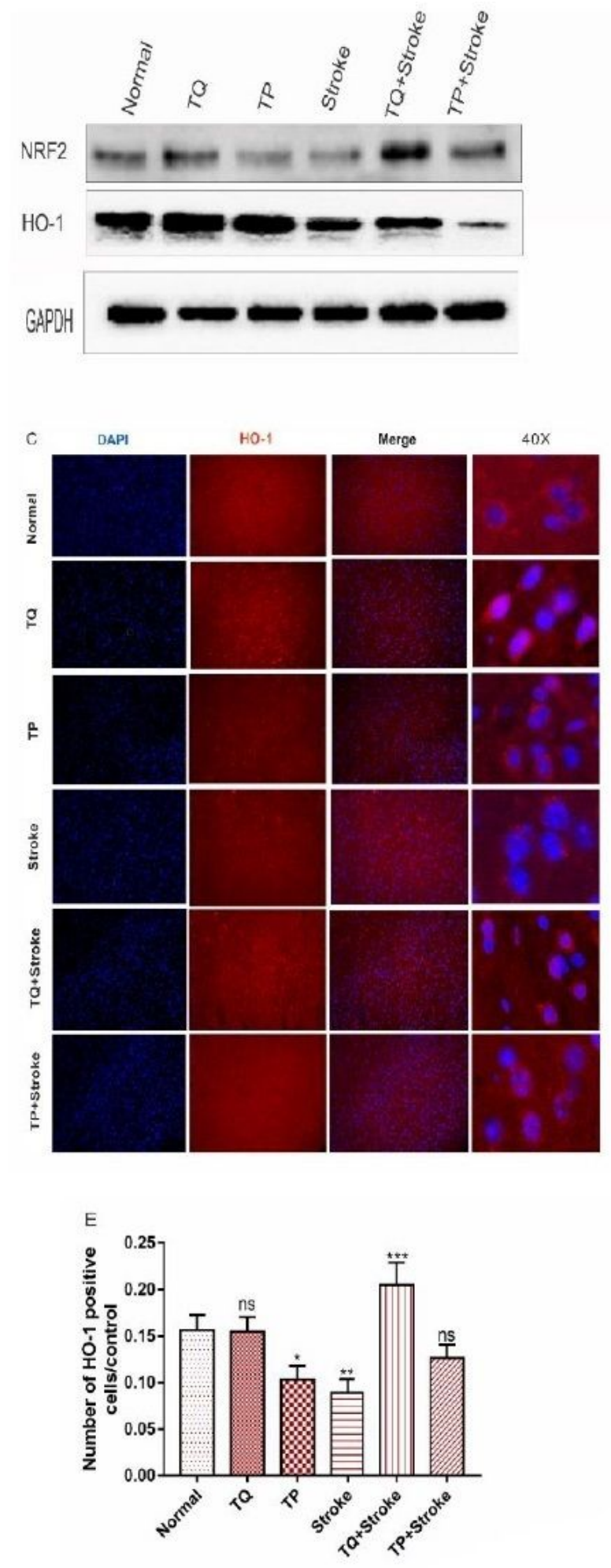
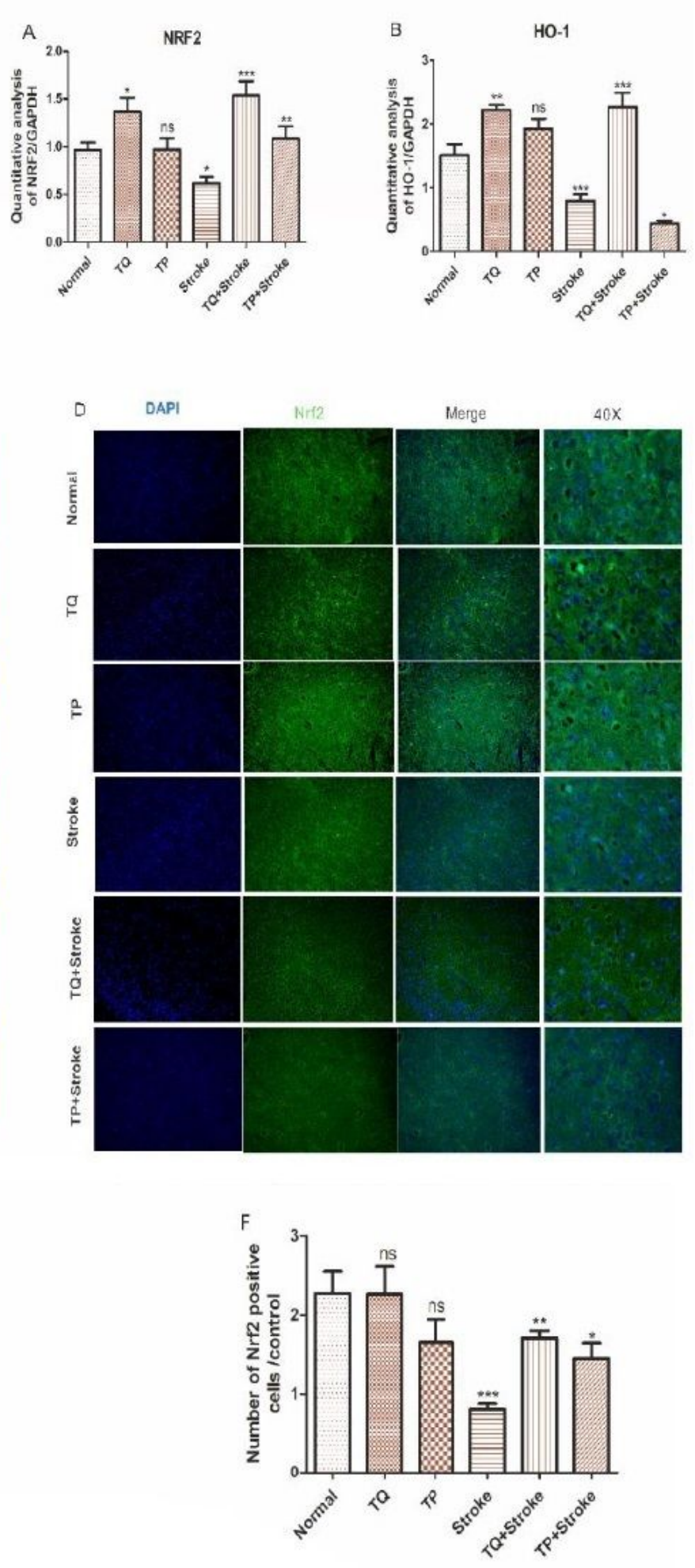

\section{Figure 3}

TQ therapy 5 days before stroke inducesantioxidant Nrf2/Ho-1 pathway. Quantification analysis of A, Nrf2. B, HO-1. Values are expressed as mean + SEM n=5. C, Immunofluorescence staining of HO-1 (red). D, Immunofluorescence staining of Nrf2(Green). E, Number of HO-1 positive cells. F, Number of Nrf2 positive cells. Stroke Vs. Normal: Nrf2 ${ }^{P}<0.05, \mathrm{HO}-1 * \star \star P<0.001, T Q+$ stroke Vs. Stroke: Nrfi2 \& HO-1 
${ }^{\star * *} \mathrm{P}<0.001, \mathrm{TP}+$ Stroke Vs. Stroke: Nrf2 ${ }^{* * P}<0.01, \mathrm{HO}-1{ }^{*} \mathrm{P}<0.05$.Values are expressed as mean $+\mathrm{SEM}$, $n=5$.
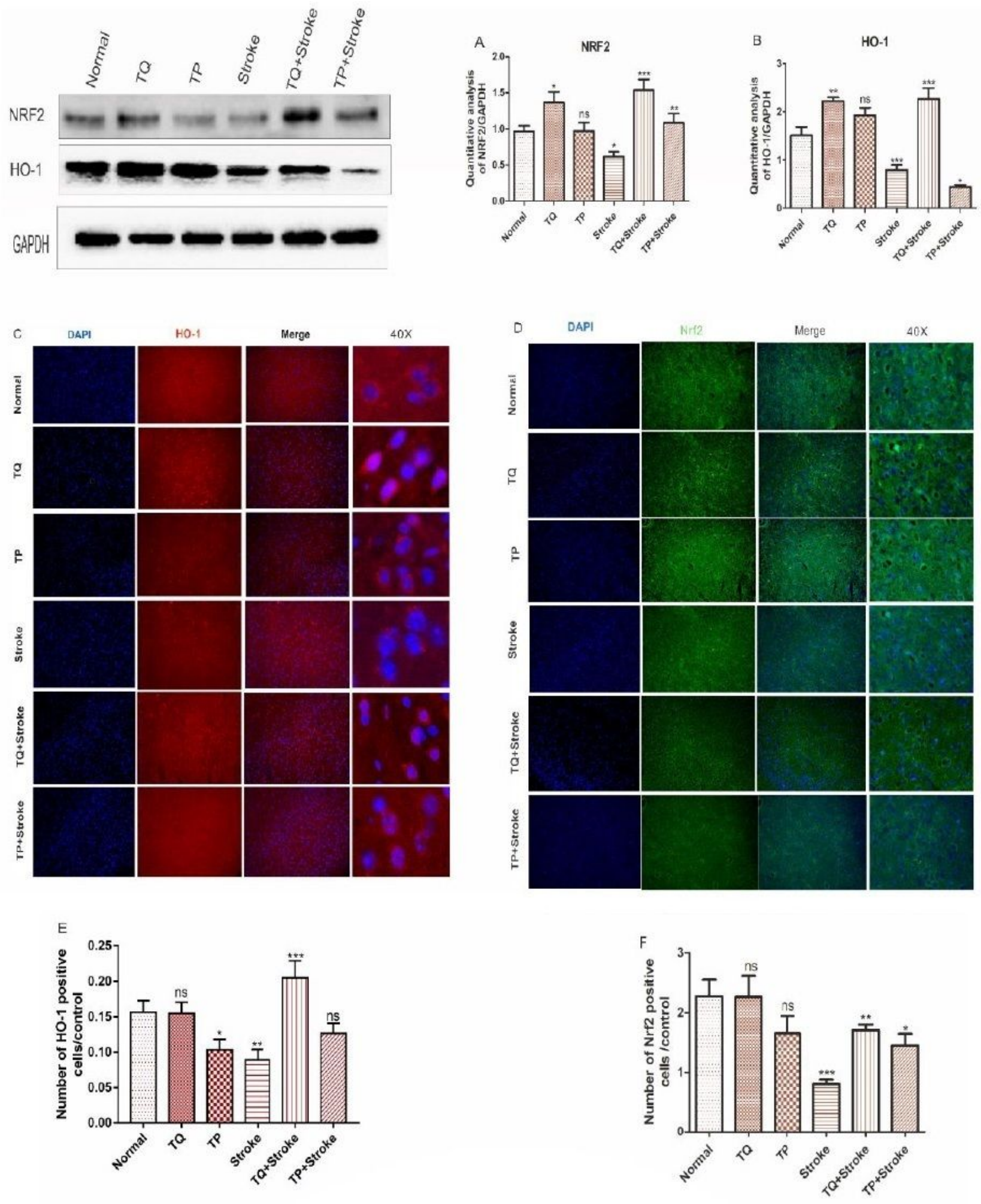

Figure 3

TQ therapy 5 days before stroke inducesantioxidant Nrf2/Ho-1 pathway. Quantification analysis of A, Nrf2. B, HO-1. Values are expressed as mean $+\mathrm{SEM} n=5$. C, Immunofluorescence staining of HO-1 (red). D, Immunofluorescence staining of Nrf2(Green). E, Number of HO-1 positive cells. F, Number of Nrf2 
positive cells. Stroke Vs. Normal: Nrf2 ${ }^{2}<<0.05, \mathrm{HO}-1 * \star * P<0.001, \mathrm{TQ}+$ stroke Vs. Stroke: Nrfi2 \& HO-1 ${ }^{\star}{ }^{*} \mathrm{P}<0.001, \mathrm{TP}+\mathrm{Str}$. $n=5$.
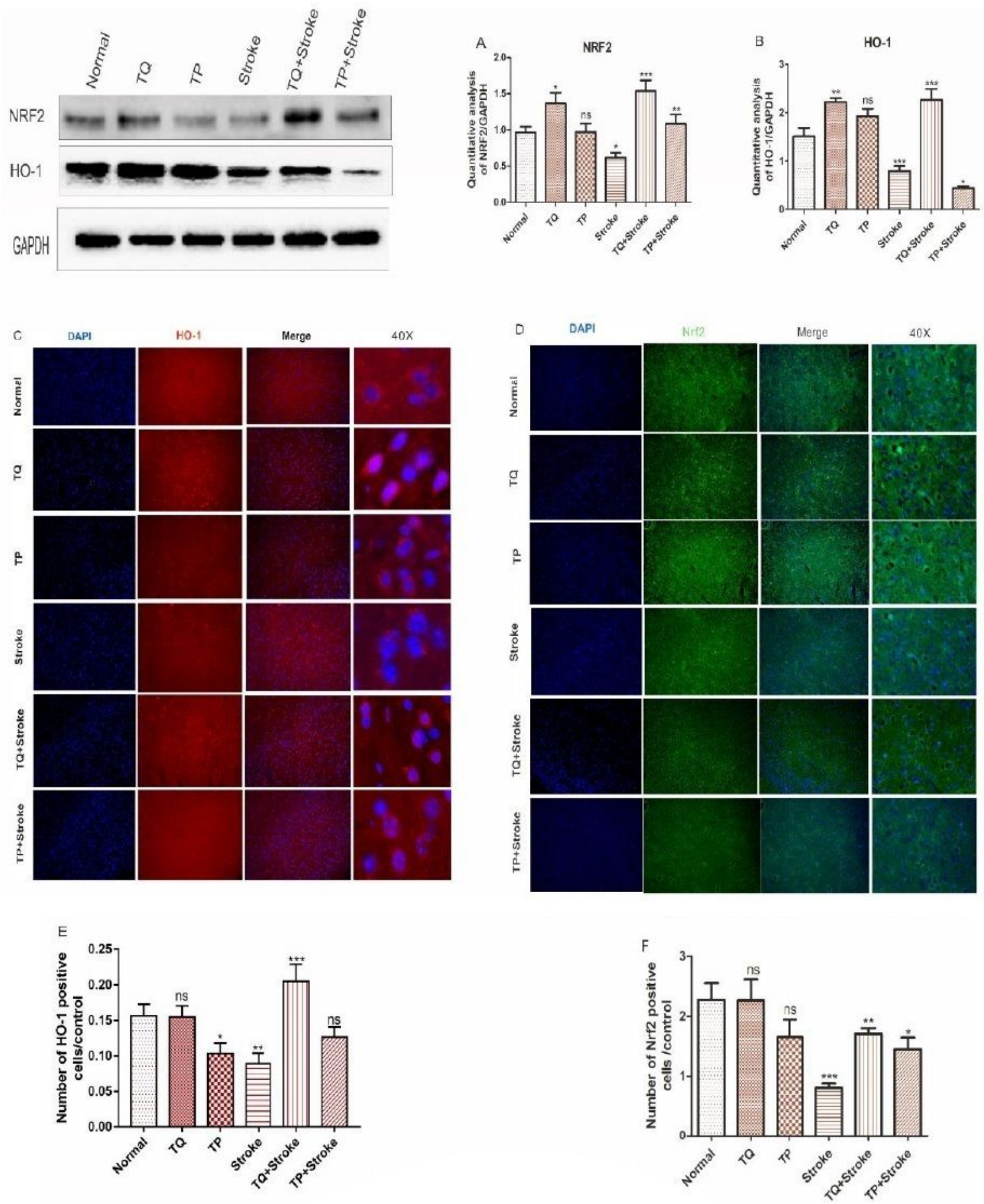

Figure 3

TQ therapy 5 days before stroke inducesantioxidant Nrf2/Ho-1 pathway. Quantification analysis of A, Nrf2. B, HO-1. Values are expressed as mean + SEM n=5. C, Immunofluorescence staining of HO-1 (red). 
D, Immunofluorescence staining of Nrf2(Green). E, Number of HO-1 positive cells. F, Number of Nrf2 positive cells. Stroke Vs. Normal: Nrf2 ${ }^{P}<0.05, \mathrm{HO}-1 * \star * P<0.001$, TQ+stroke Vs. Stroke: Nrfi2 \& HO-1 ${ }^{\star} * * \mathrm{P}<0.001, \mathrm{TP}+\mathrm{Str}$ ke Vs. Stroke: Nrf2 ${ }^{*} \mathrm{P}<0.01, \mathrm{HO}-1{ }^{*} \mathrm{P}<0.05$.Values are expressed as mean $+\mathrm{SEM}$, $n=5$.

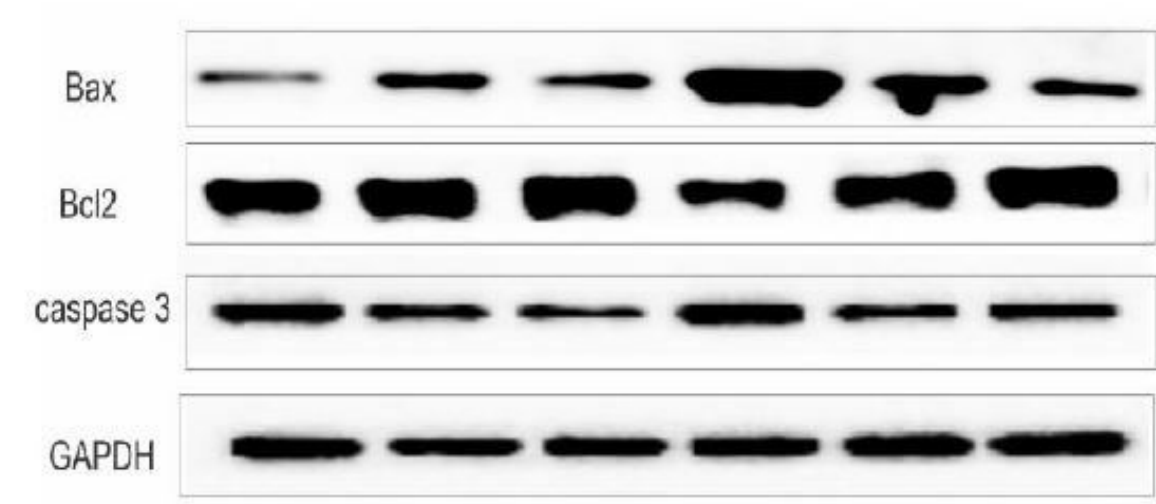

B

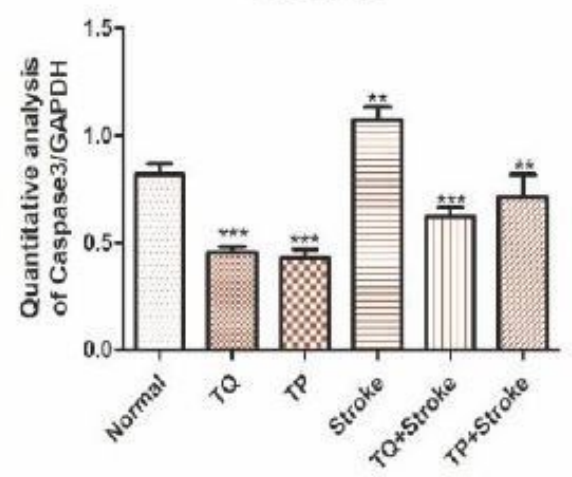

A

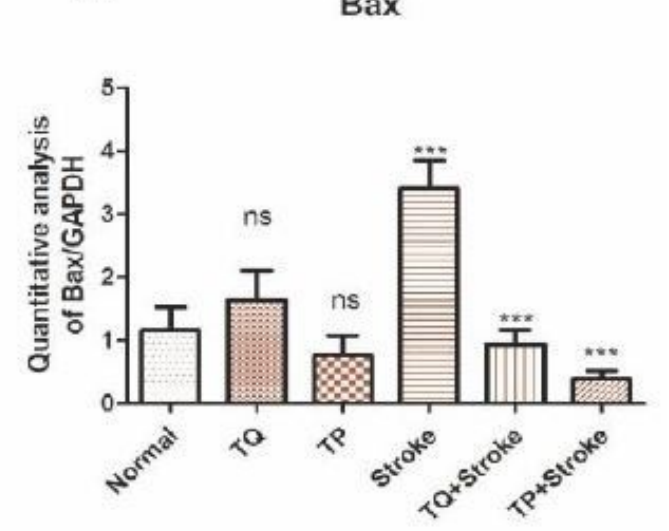

B

Caspase 3

C

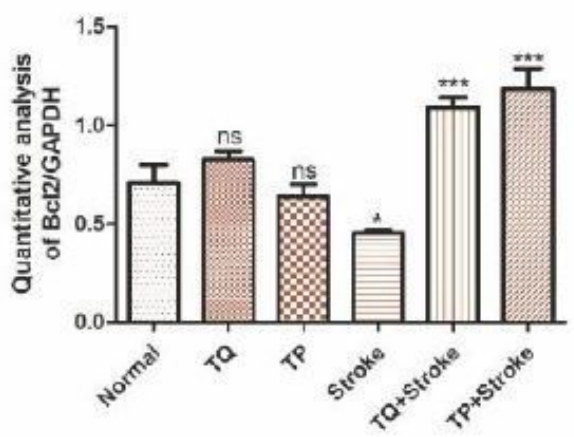

Figure 4

TQ therapy 5 days before stroke reduce the neuronal cells death. Western blot results of $\mathrm{Bax}, \mathrm{Bcl} 2$, Caspase 3 and GADPH. Quantification analysis of (A)Bax, (B) Bcl2, (C) Caspase 3Stroke Vs. normal; Bax \& caspase ${ }^{* \star *} \mathrm{P}<0.001 \mathrm{Bcl} 2{ }^{*} \mathrm{P}<0.05, \mathrm{TQ}+$ stroke Vs. Stroke: Bax, caspase3 \&Bcl2 ${ }^{\star \star \star} \mathrm{P}<0.001, \mathrm{~T}+\mathrm{Stroke}$ vs Stroke; $B a x \& B c l 2 * * * P<0.001$, caspase $3 * * P<0.01$. All values are expressed as mean $+S E M, n=5$. 


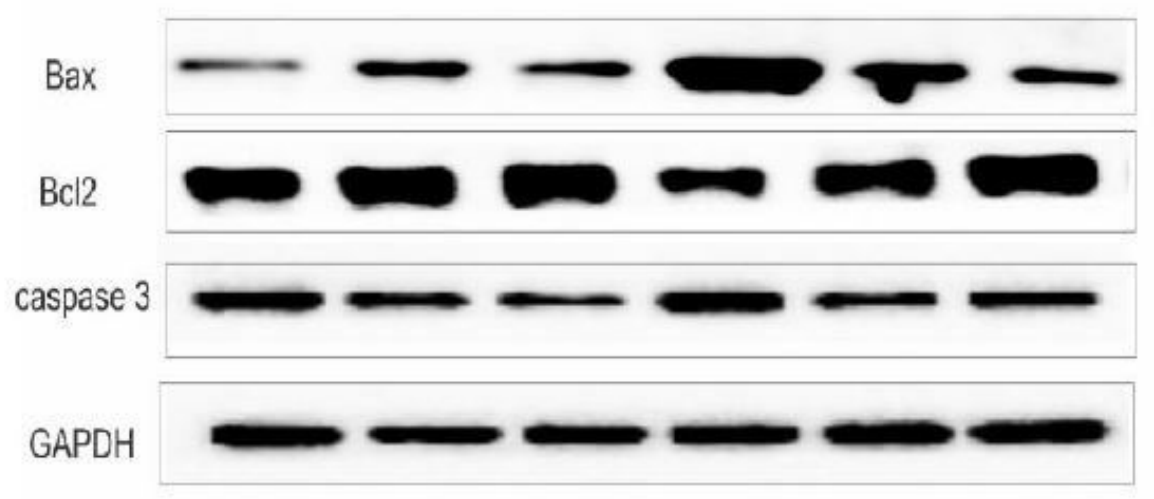

B

Caspase 3

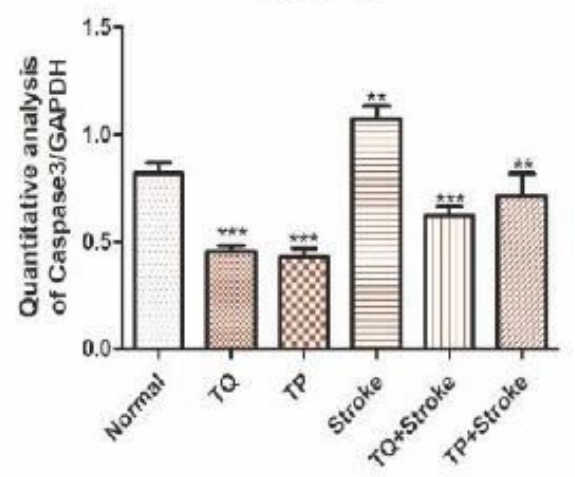

A

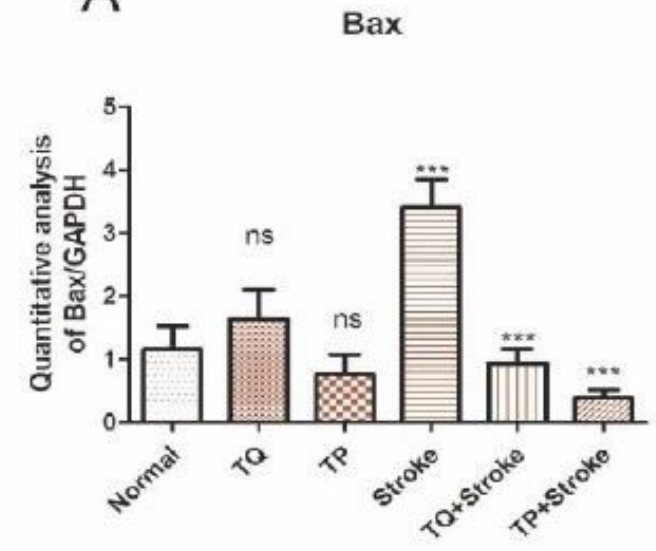

C

$\mathrm{Bcl} 2$

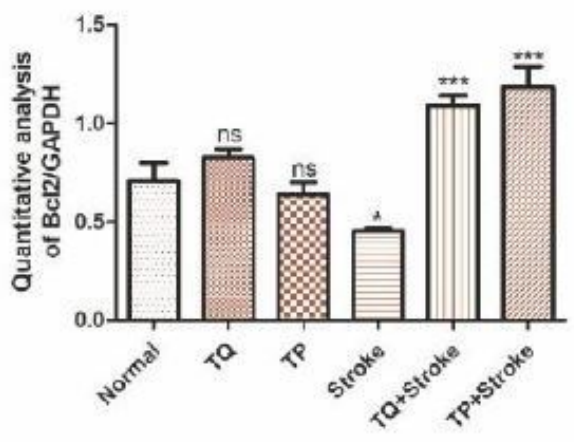

\section{Figure 4}

TQ therapy 5 days before stroke reduce the neuronal cells death. Western blot results of Bax, Bcl2, Caspase 3 and GADPH. Quantification analysis of (A)Bax, (B) Bcl2, (C) Caspase 3Stroke Vs. normal; Bax \& caspase ${ }^{\star \star *} \mathrm{P}<0.001 \mathrm{Bcl} 2{ }^{*} \mathrm{P}<0.05, \mathrm{TQ}+$ stroke Vs. Stroke: Bax, caspase3 \&Bcl2***P<0.001,TP+Stroke vs Stroke; $B a x \& B c l 2 * \star * P<0.001$, caspase3 ${ }^{* * P}<0.01$. All values are expressed as mean $+S E M, n=5$. 


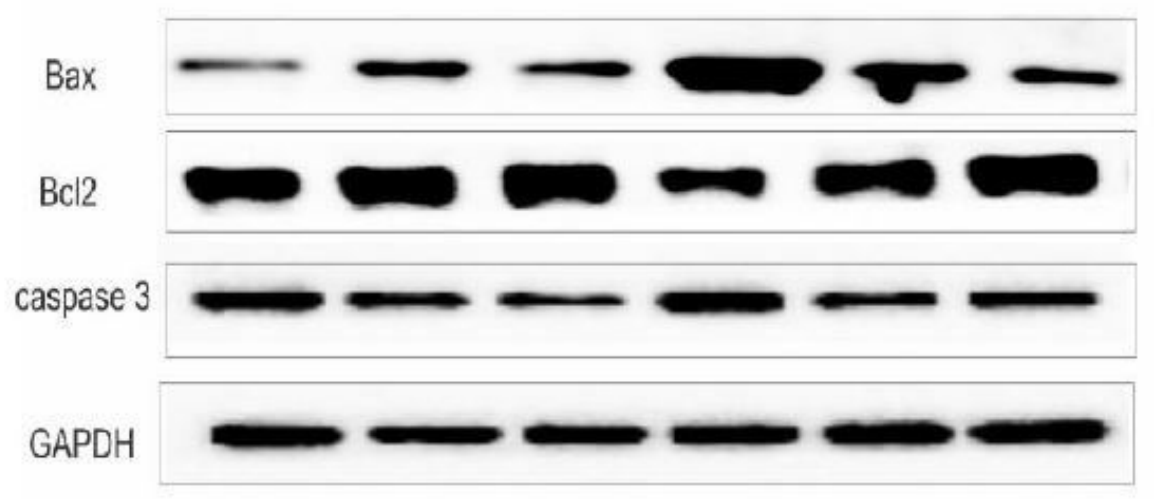

B

Caspase 3

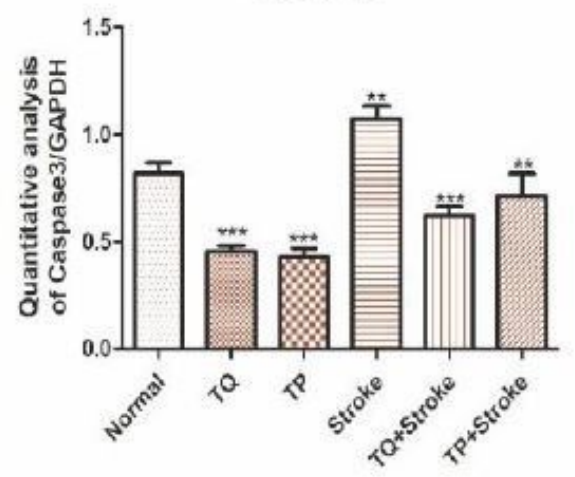

A

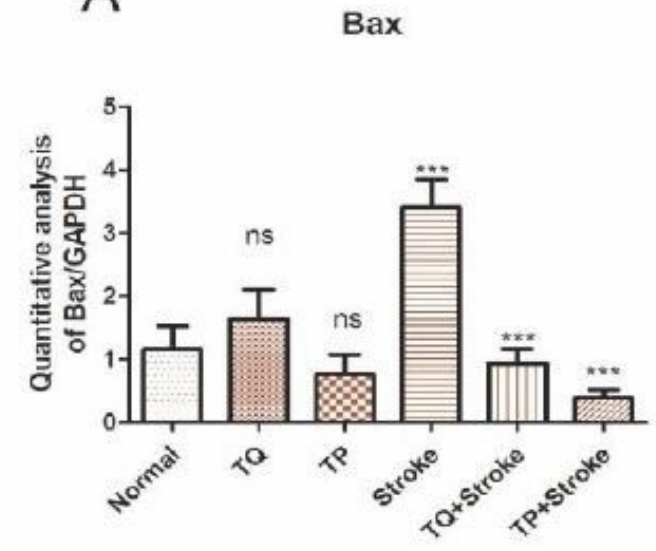

C

$\mathrm{Bcl} 2$

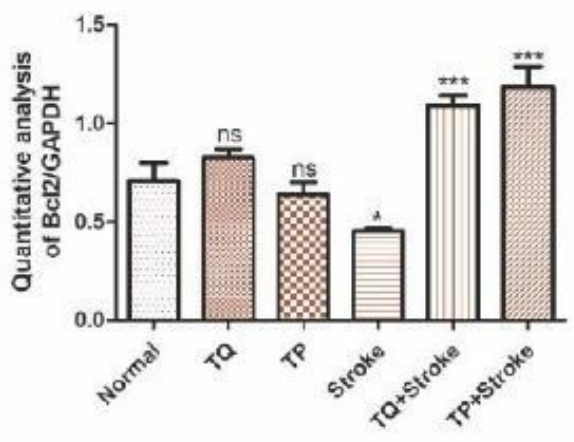

\section{Figure 4}

TQ therapy 5 days before stroke reduce the neuronal cells death. Western blot results of Bax, Bcl2, Caspase 3 and GADPH. Quantification analysis of (A)Bax, (B) Bcl2, (C) Caspase 3Stroke Vs. normal; Bax \& caspase ${ }^{\star \star *} \mathrm{P}<0.001 \mathrm{Bcl} 2{ }^{*} \mathrm{P}<0.05, \mathrm{TQ}+$ stroke Vs. Stroke: Bax, caspase3 \&Bcl2***P<0.001,TP+Stroke vs Stroke; $B a x \& B c l 2 * \star * P<0.001$, caspase3 ${ }^{* * P}<0.01$. All values are expressed as mean $+S E M, n=5$. 

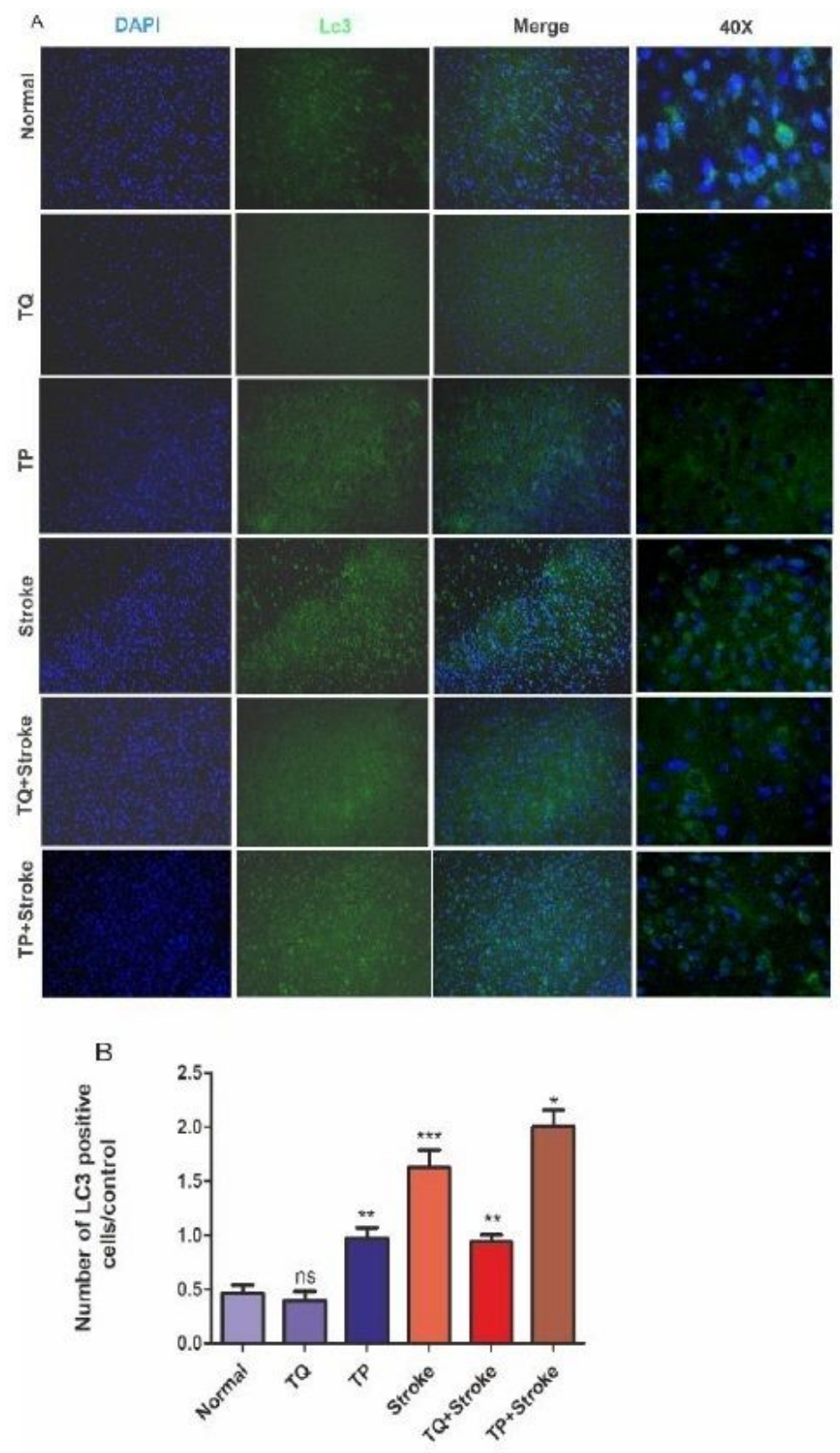
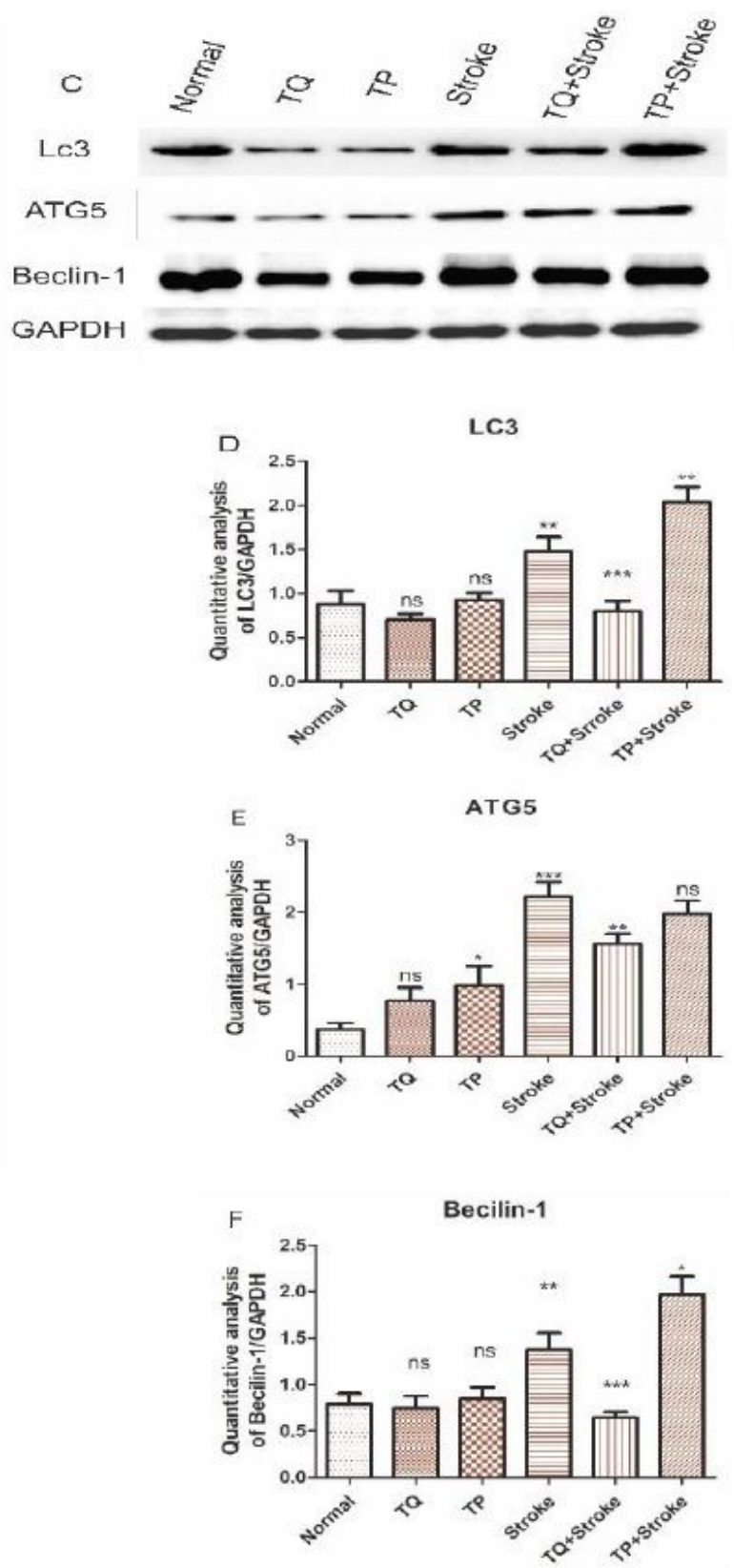

\section{Figure 5}

TQ therapy 5 days before stroke can alleviate the excessive autophagy response. (A) Immunofluorescence staining of LC3, (B) Number of LC3 positive cells, (C) Western blot results of LC3, ATG5, Beclia1, and GADPH. Quantification analysis of (D)LC3, (E) ATGS, (F) Beclia1. Values are expressed as mean + SEM $n=5$. Stroke Vs. Normal: LC3\& Beclin $-1{ }^{\star *} P<0.01, A T G 5 * \star * P<0.001, T Q+$ Stroke Vs. Stroke: LC3\& Beclin $-1{ }^{\star *} P<0.001$, ATG5 ${ }^{\star *} P<0.01$, TP+ Stroke Vs. Stroke: LC3 ${ }^{\star \star P}<0.01$, Beclin-1 ${ }^{*} \mathrm{P}<0.05$, ATG5 $\mathrm{P}=\mathrm{ns}$. 

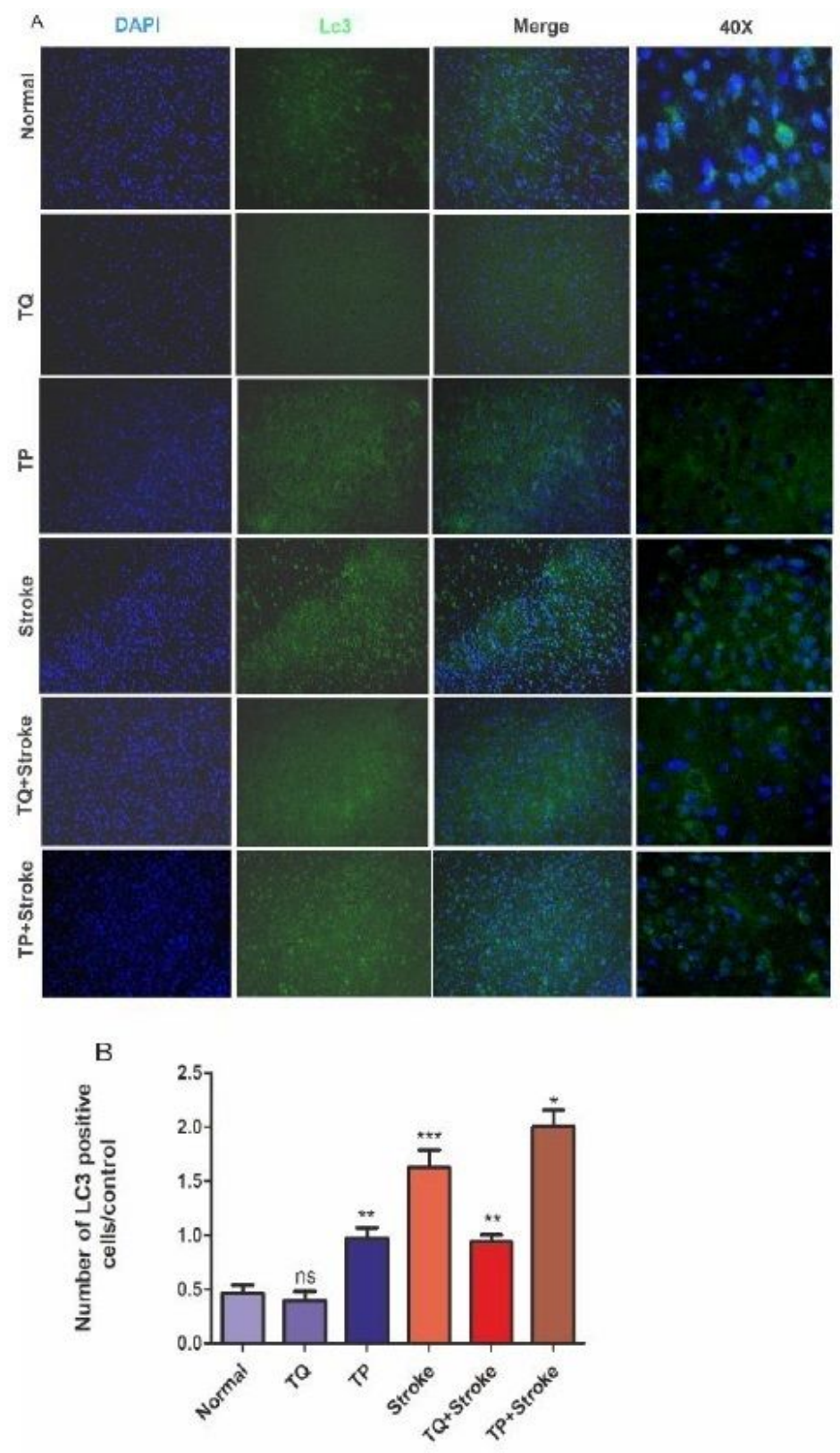
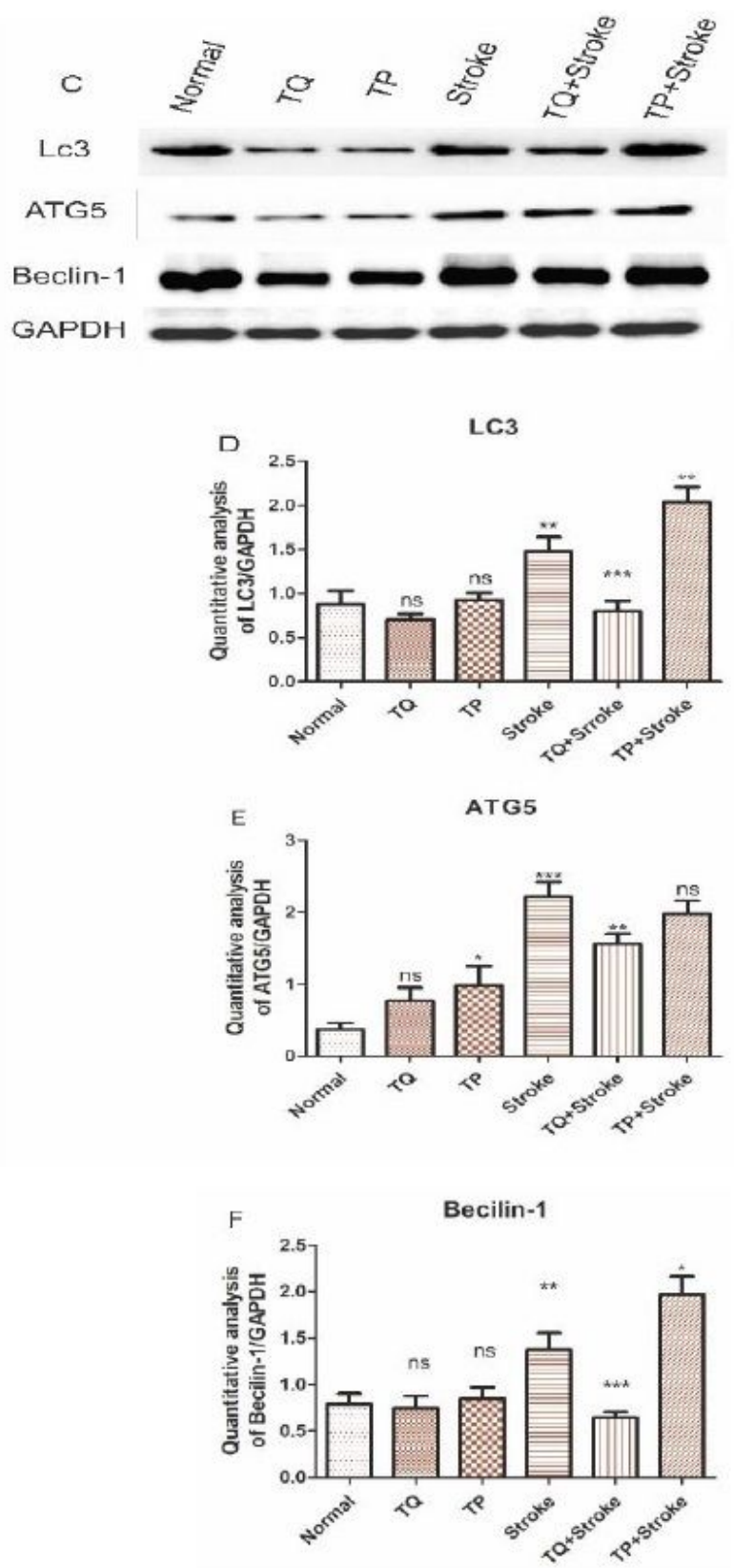

\section{Figure 5}

TQ therapy 5 days before stroke can alleviate the excessive autophagy response. (A) Immunofluorescence staining of LC3, (B) Number of LC3 positive cells, (C) Western blot results of LC3, ATG5, Beclia1, and GADPH. Quantification analysis of (D)LC3, (E) ATGS, (F) Beclia1. Values are expressed as mean + SEM $n=5$. Stroke Vs. Normal: LC3\& Beclin $-1{ }^{\star *} P<0.01, A T G 5 * \star * P<0.001, T Q+$ Stroke Vs. Stroke: LC3\& Beclin $-1{ }^{\star *} P<0.001$, ATG5 ${ }^{\star *} P<0.01$, TP+ Stroke Vs. Stroke: LC3 ${ }^{\star \star P}<0.01$, Beclin-1 ${ }^{*} \mathrm{P}<0.05$, ATG5 $\mathrm{P}=\mathrm{ns}$. 

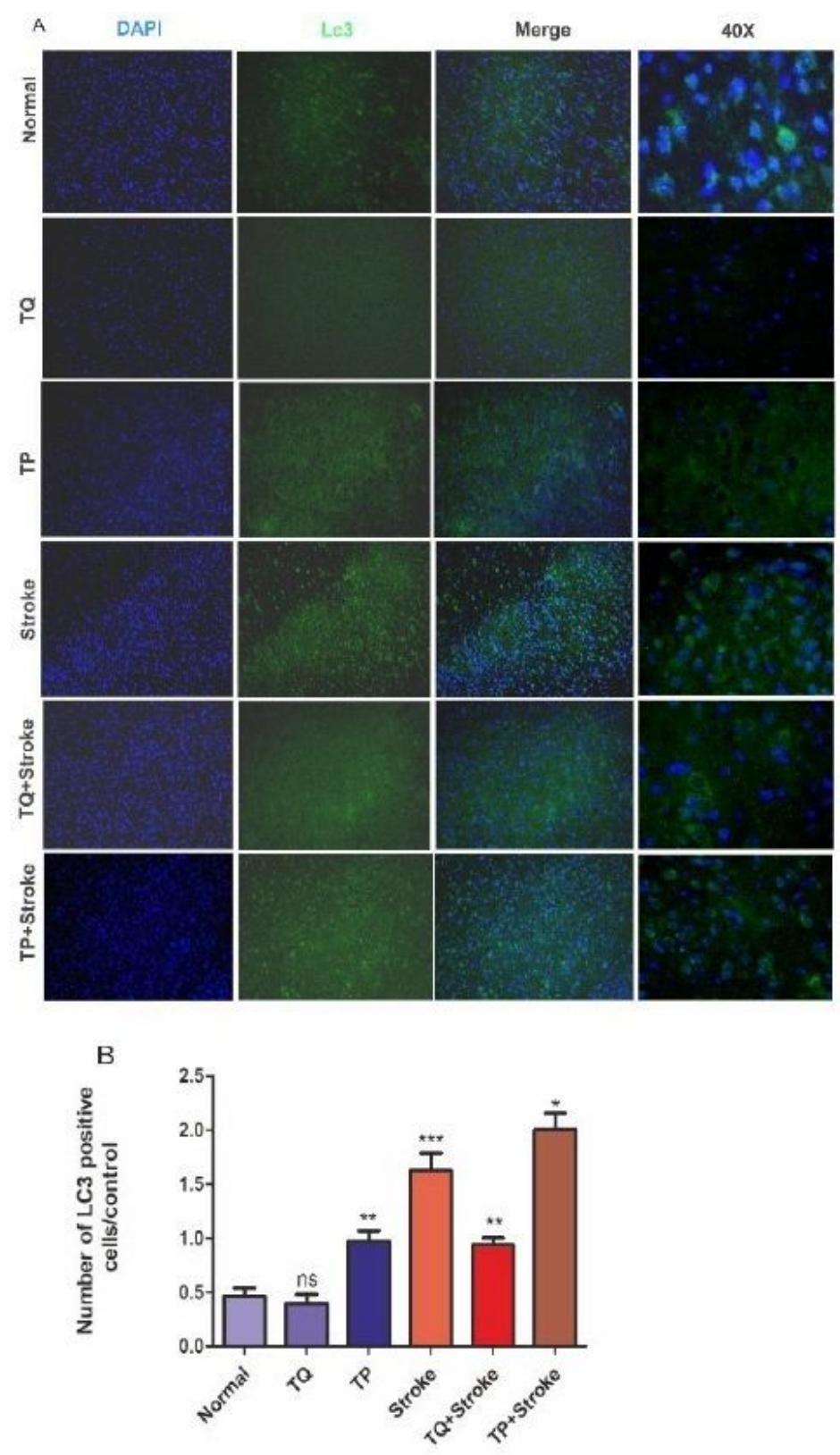
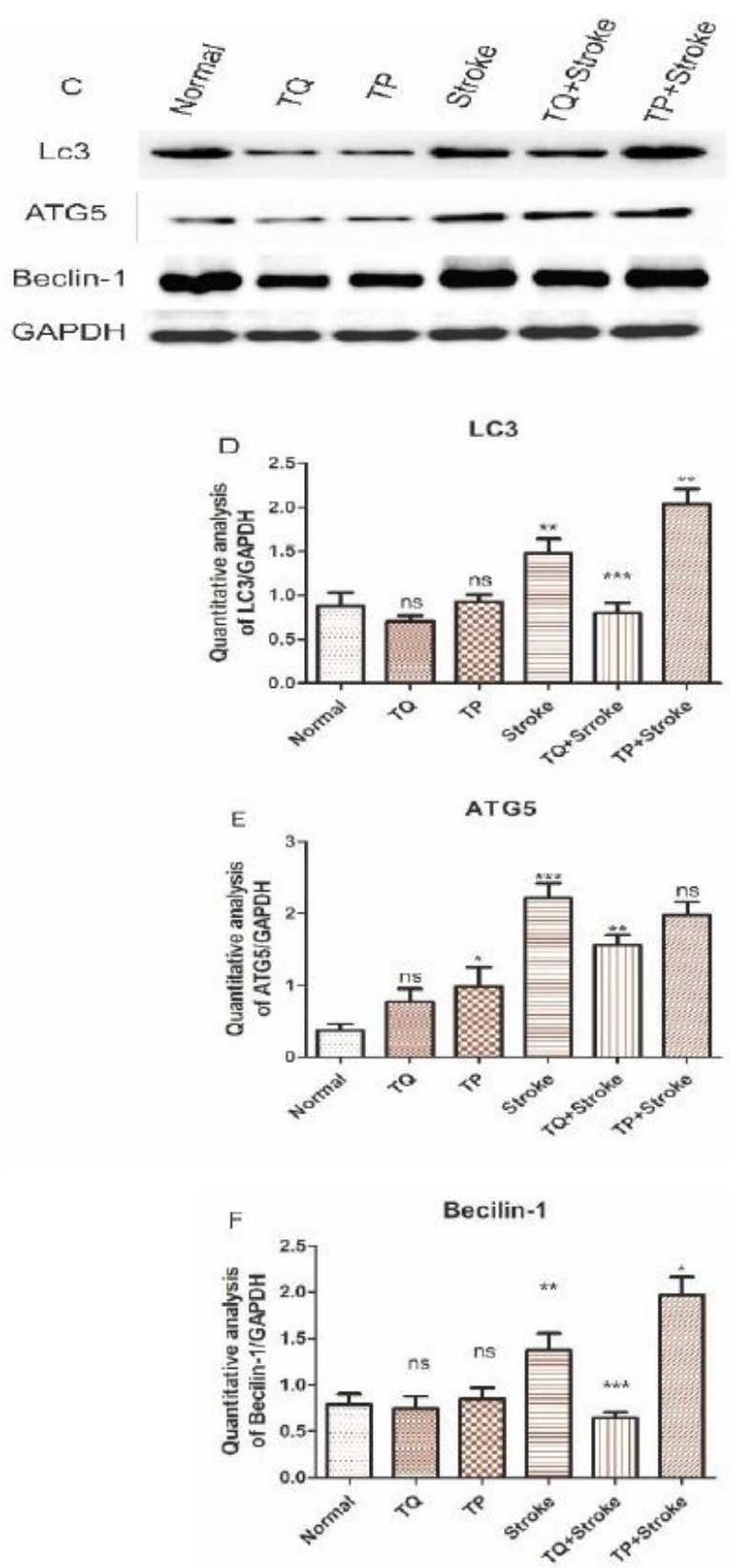

\section{Figure 5}

TQ therapy 5 days before stroke can alleviate the excessive autophagy response. (A) Immunofluorescence staining of LC3, (B) Number of LC3 positive cells, (C) Western blot results of LC3, ATG5, Beclia1, and GADPH. Quantification analysis of (D)LC3, (E) ATGS, (F) Beclia1. Values are expressed as mean + SEM $n=5$. Stroke Vs. Normal: LC3\& Beclin $-1{ }^{\star *} P<0.01, A T G 5 * \star * P<0.001, T Q+$ Stroke Vs. Stroke: LC3\& Beclin $-1{ }^{\star *} P<0.001$, ATG5 ${ }^{\star *} P<0.01$, TP+ Stroke Vs. Stroke: LC3 ${ }^{\star \star P}<0.01$, Beclin-1 ${ }^{*} \mathrm{P}<0.05$, ATG5 $\mathrm{P}=\mathrm{ns}$. 

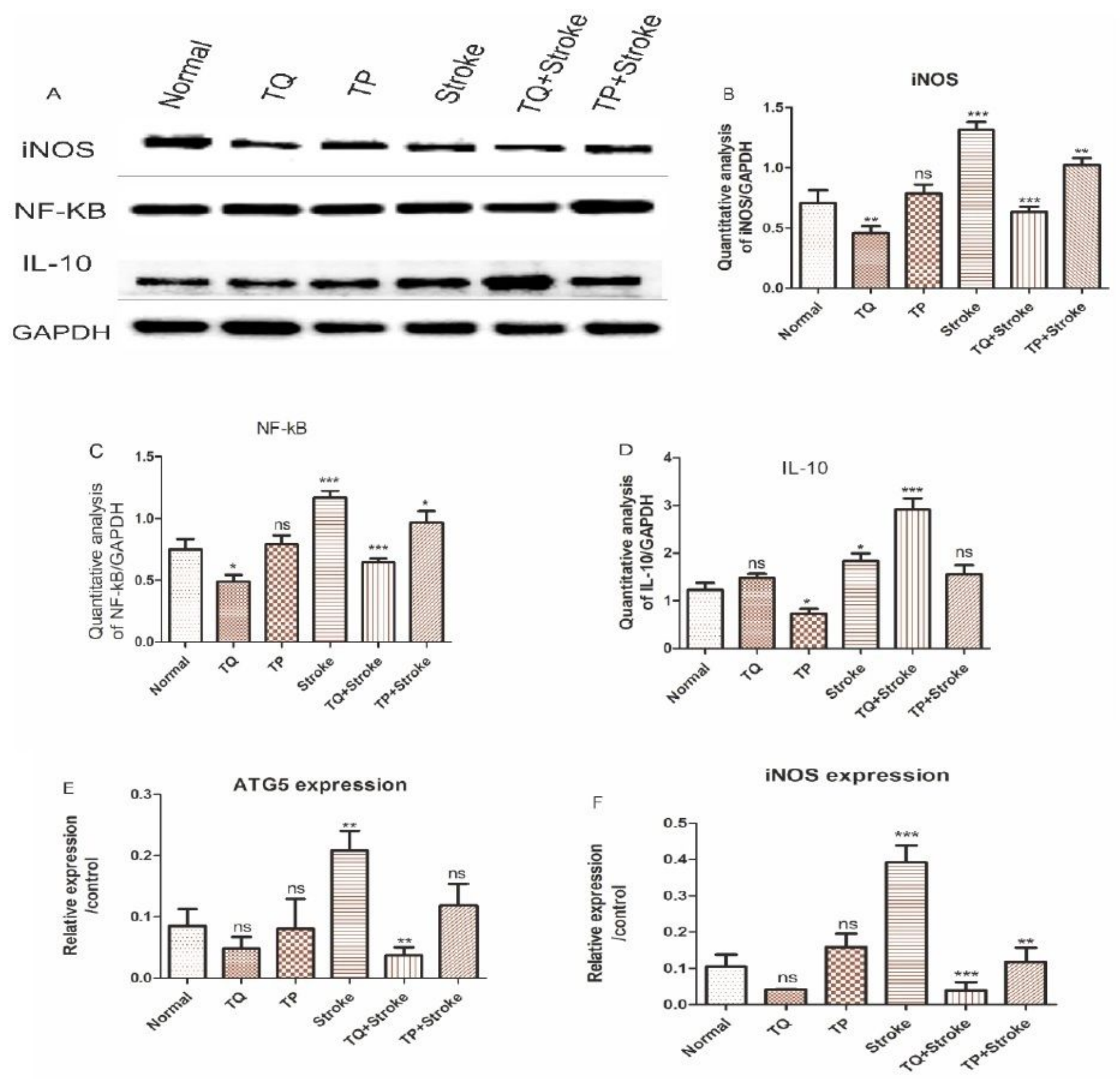

\section{Figure 6}

TQ therapy significantly alleviate inflammation via inhibiting NF - kB/iNOS and inducing IL-10. (A) Western blot results of INOS, NF-kB, IL-10 and GADPH. Quantification analysis of (B) iNOS (C) NF -kB (D) IL-10 (E) ATG5 gene analysis, (F) iNOS gene analysis ; Stroke Vs. Normal: NF -kB \& INOS***P<0.001, IL-10

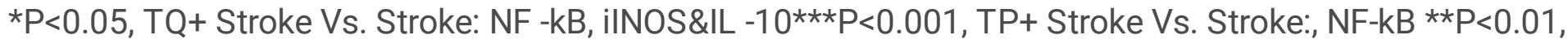
INOS $* \mathrm{P}<0.05$ and IL-10 $\mathrm{P}=\mathrm{ns}$. All values are expressed as mean $+\mathrm{SEM}, \mathrm{n}=5$. 

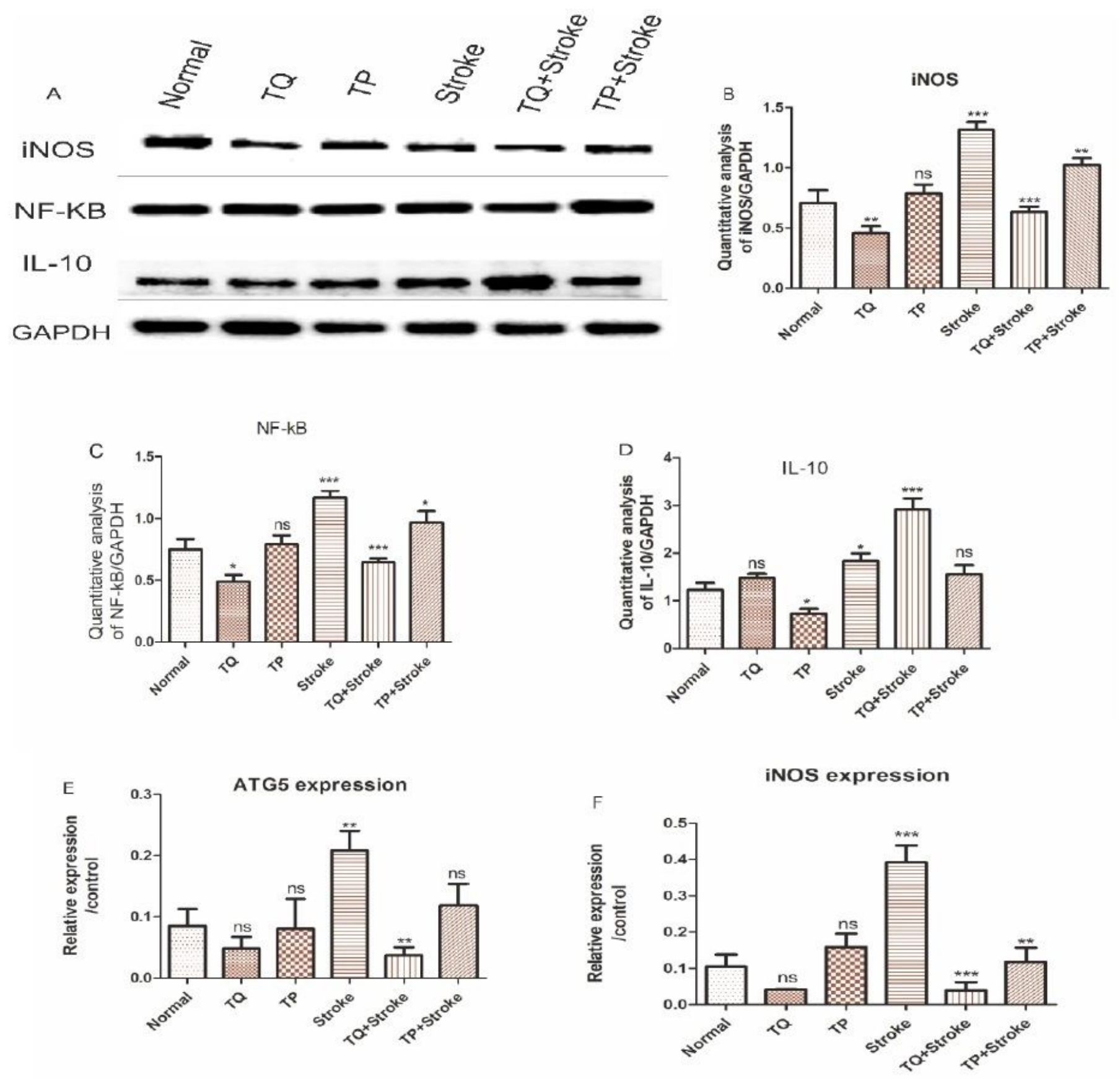

\section{Figure 6}

TQ therapy significantly alleviate inflammation via inhibiting NF - kB/iNOS and inducing IL-10. (A) Western blot results of INOS, NF-kB, IL-10 and GADPH. Quantification analysis of (B) iNOS (C) NF -kB (D) IL-10 (E) ATG5 gene analysis, (F) iNOS gene analysis ; Stroke Vs. Normal: NF -kB \& INOS***P<0.001, IL-10

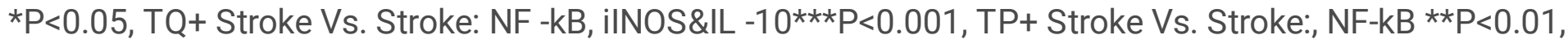
INOS $* \mathrm{P}<0.05$ and IL-10 $\mathrm{P}=\mathrm{ns}$. All values are expressed as mean $+\mathrm{SEM}, \mathrm{n}=5$. 

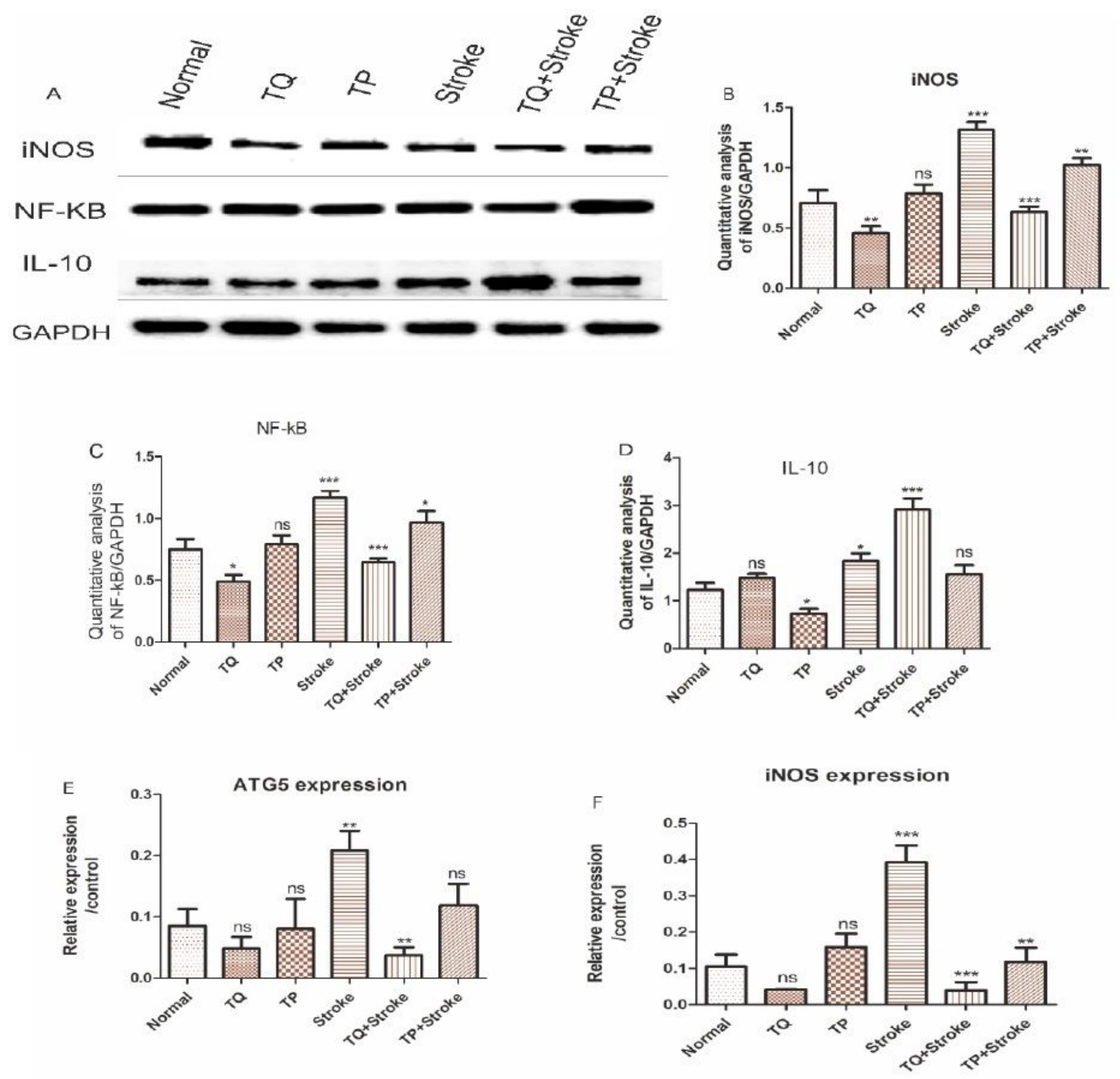

\section{Figure 6}

TQ therapy significantly alleviate inflammation via inhibiting NF - kB/iNOS and inducing IL-10. (A) Western blot results of INOS, NF-kB, IL-10 and GADPH. Quantification analysis of (B) iNOS (C) NF -kB (D) IL-10 (E) ATG5 gene analysis, (F) iNOS gene analysis ; Stroke Vs. Normal: NF -kB \& INOS***P<0.001, IL-10

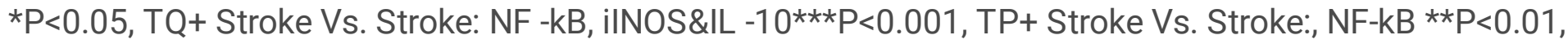
INOS $* \mathrm{P}<0.05$ and IL-10 $\mathrm{P}=\mathrm{ns}$. All values are expressed as mean $+\mathrm{SEM}, \mathrm{n}=5$. 

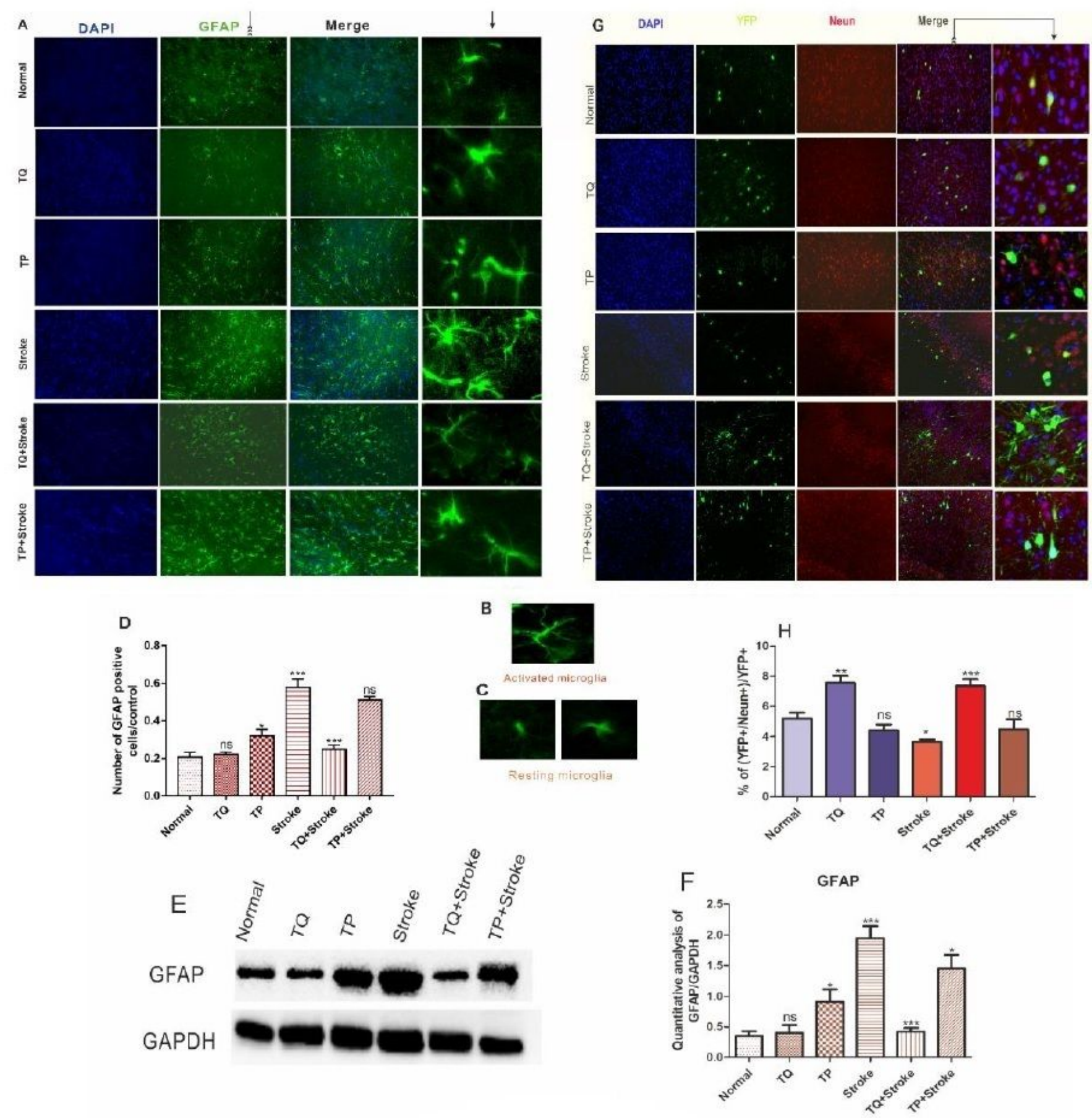

\section{Figure 7}

TQ therapy improves neurogenesis and _ inhibiting the inflammatory activation of astrocyte., (A) Immunofluorescence staining of GFAP (green), (B) activated microglia, (C) Resting microglia, (D) Number of GFAP positive cells. (E) Western blot results of GFAP and GADPH. Quantification analysis 6(F) GFAP (G) YFP (Green) co -labeling NeuN (Red). (H) The number of YFP reporter cells. All values are expressed aS mean + SEM, $n=5$ Stroke Vs. Normal: GFAP *** $P<0.001$ YFP reporter cells ${ }^{*} P<0.05, T Q+$ Stroke Vs. Stroke: GFAP ${ }^{* \star *} P<0.001$, YFP reporter cells ${ }^{* \star * P}<0.001$, TP+ Stroke Vs. Stroke: GFAP $* P<0.05$, YFP reporter cells $\mathrm{P}=\mathrm{ns}$. 

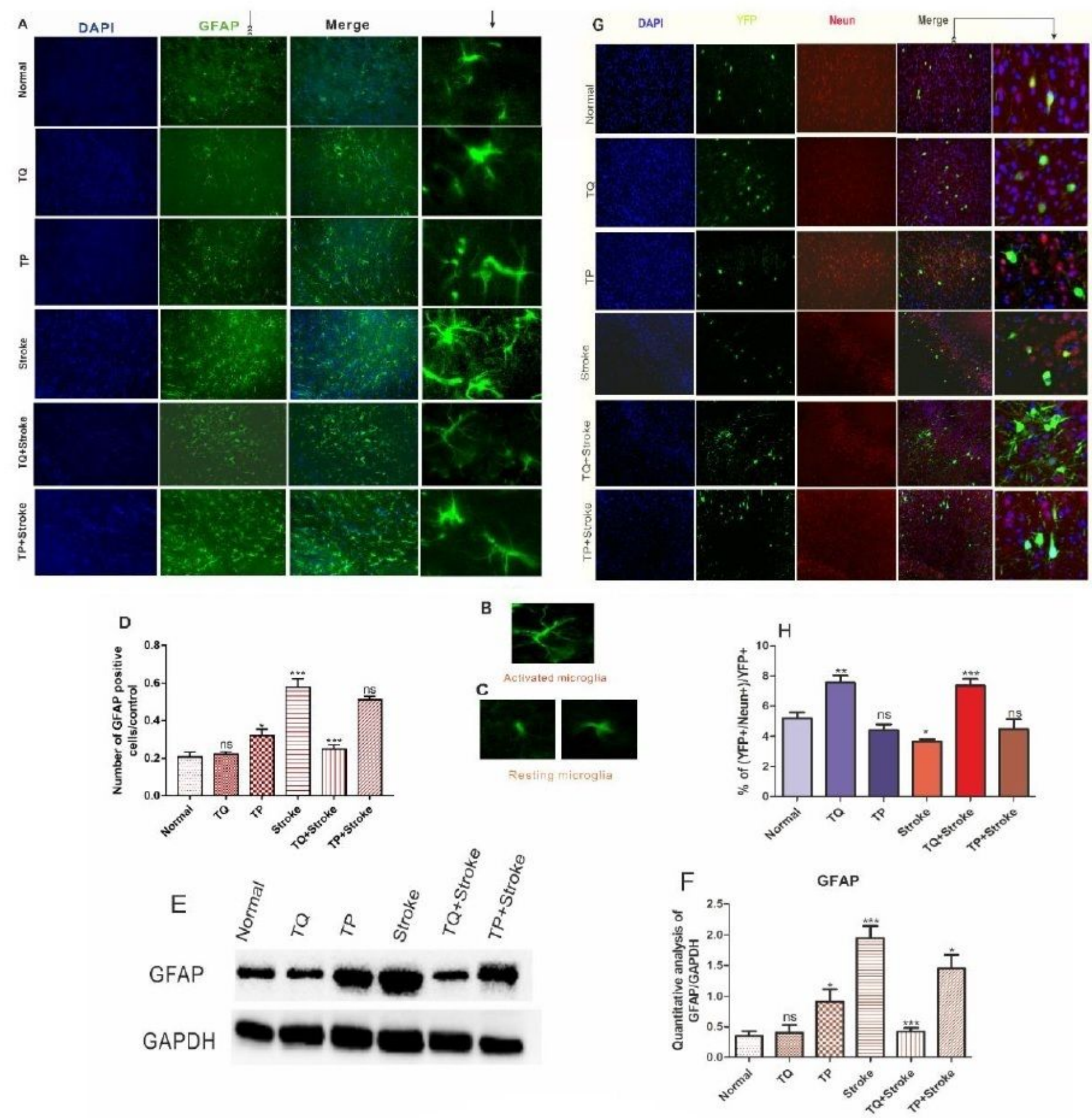

\section{Figure 7}

TQ therapy improves neurogenesis and _ inhibiting the inflammatory activation of astrocyte., (A) Immunofluorescence staining of GFAP (green), (B) activated microglia, (C) Resting microglia, (D) Number of GFAP positive cells. (E) Western blot results of GFAP and GADPH. Quantification analysis 6(F) GFAP (G) YFP (Green) co -labeling NeuN (Red). (H) The number of YFP reporter cells. All values are expressed aS mean + SEM, $n=5$ Stroke Vs. Normal: GFAP *** $P<0.001$ YFP reporter cells ${ }^{*} P<0.05, T Q+$ Stroke Vs. Stroke: GFAP ${ }^{* \star *} P<0.001$, YFP reporter cells ${ }^{* \star * P}<0.001$, TP+ Stroke Vs. Stroke: GFAP $* P<0.05$, YFP reporter cells $\mathrm{P}=\mathrm{ns}$. 

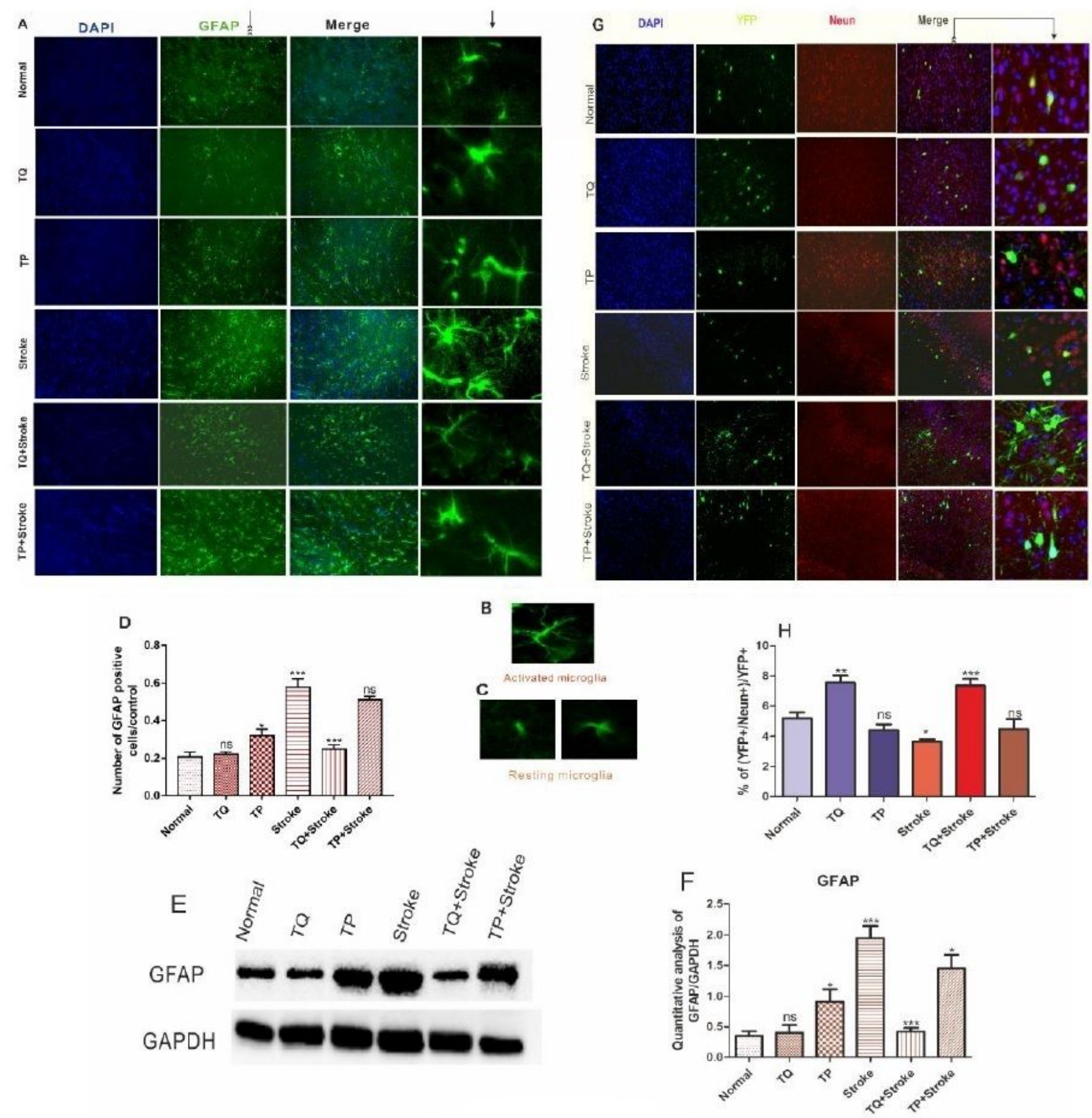

\section{Figure 7}

TQ therapy improves neurogenesis and _ inhibiting the inflammatory activation of astrocyte., (A) Immunofluorescence staining of GFAP (green), (B) activated microglia, (C) Resting microglia, (D) Number of GFAP positive cells. (E) Western blot results of GFAP and GADPH. Quantification analysis 6(F) GFAP (G) YFP (Green) co -labeling NeuN (Red). (H) The number of YFP reporter cells. All values are expressed aS mean + SEM, $n=5$ Stroke Vs. Normal: GFAP *** $P<0.001$ YFP reporter cells ${ }^{*} P<0.05, T Q+$ Stroke Vs. Stroke: GFAP ${ }^{* \star *} P<0.001$, YFP reporter cells ${ }^{* \star * P}<0.001$, TP+ Stroke Vs. Stroke: GFAP $* P<0.05$, YFP reporter cells $\mathrm{P}=\mathrm{ns}$. 


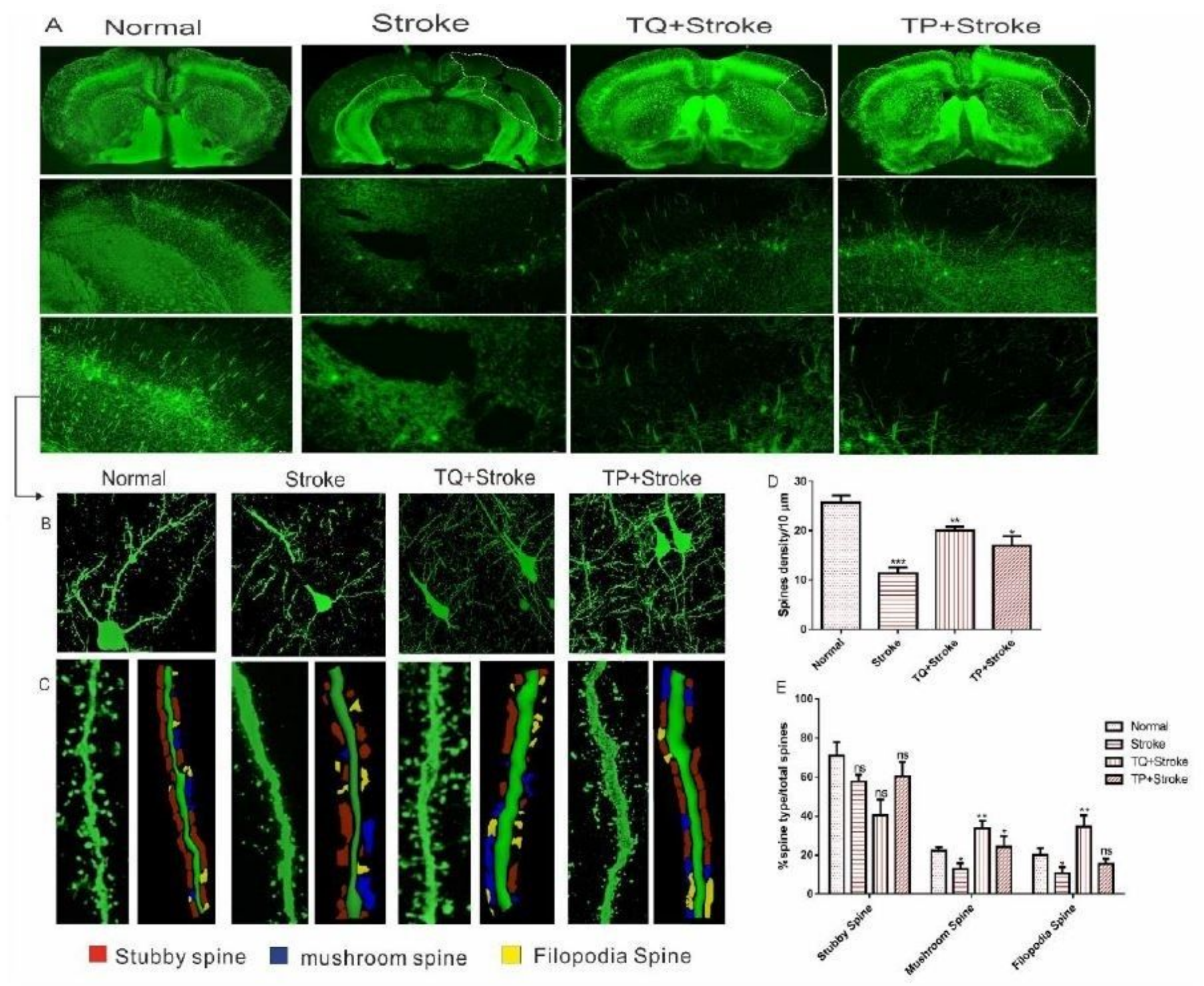

Figure 8

TQ therapy elevates the dendrite spines density and attenuates the neuron damaged area after stroke. (A) YFP slices observation for the stroked area. $(B, C)$ Confocal microscope images of neurons and spines

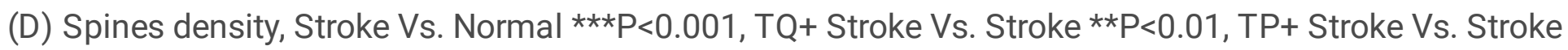
${ }^{*} \mathrm{P}<0.05$ (E) \%Spines type, Mushroom type; Stroke Vs. Normal ${ }^{*} \mathrm{P}<0.05, T Q+$ Stroke Vs. Stroke ${ }^{* \star} P<0.01$, $T P+$ Stroke Vs. Stroke ${ }^{*} P<0.05$, Filopodia type; Stroke Vs. Normal ${ }^{*}<<0.05, T Q+S t r o k e ~ V s$. Stroke $\star \star P<0.01, T P+$ Stroke Vs. Stroke $P=n s$. All values are expressed as mean $+S E M, n=5$. 


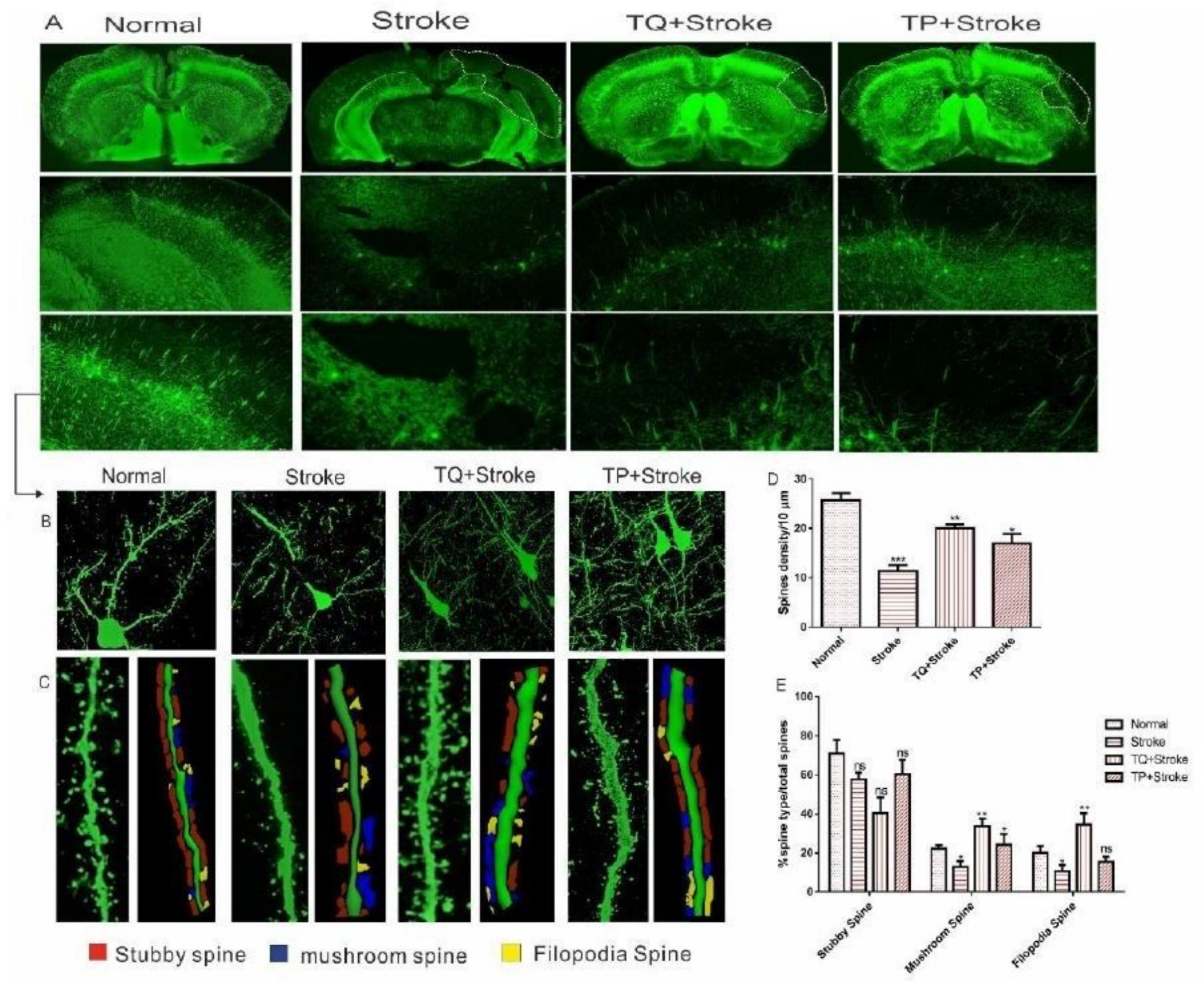

Figure 8

TQ therapy elevates the dendrite spines density and attenuates the neuron damaged area after stroke. (A) YFP slices observation for the stroked area. $(B, C)$ Confocal microscope images of neurons and spines

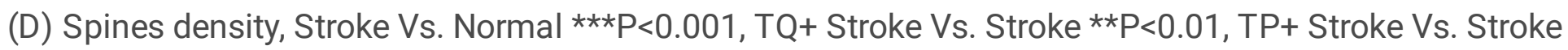
${ }^{*} \mathrm{P}<0.05$ (E) \%Spines type, Mushroom type; Stroke Vs. Normal ${ }^{*} \mathrm{P}<0.05, T Q+$ Stroke Vs. Stroke ${ }^{* \star} P<0.01$, $T P+$ Stroke Vs. Stroke ${ }^{*} P<0.05$, Filopodia type; Stroke Vs. Normal ${ }^{*}<<0.05, T Q+S t r o k e ~ V s$. Stroke $\star \star P<0.01, T P+$ Stroke Vs. Stroke $P=n s$. All values are expressed as mean $+S E M, n=5$. 


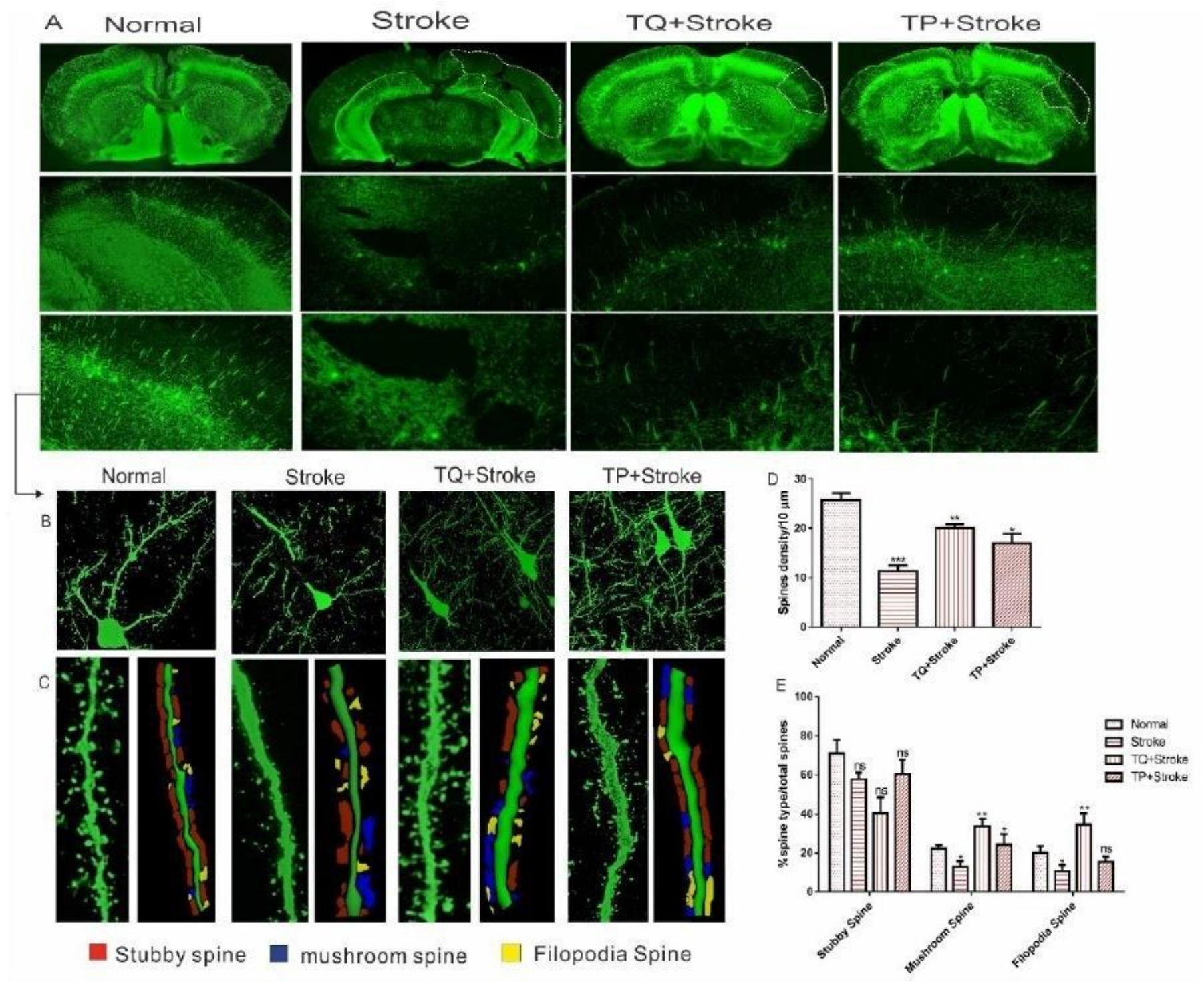

Figure 8

TQ therapy elevates the dendrite spines density and attenuates the neuron damaged area after stroke. (A) YFP slices observation for the stroked area. $(B, C)$ Confocal microscope images of neurons and spines

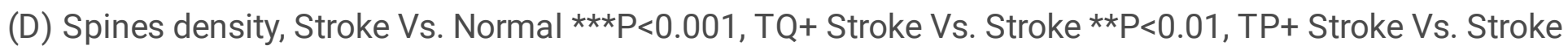
${ }^{*} \mathrm{P}<0.05$ (E) \%Spines type, Mushroom type; Stroke Vs. Normal ${ }^{*} \mathrm{P}<0.05, T Q+$ Stroke Vs. Stroke ${ }^{* \star} P<0.01$, $T P+$ Stroke Vs. Stroke ${ }^{*} P<0.05$, Filopodia type; Stroke Vs. Normal ${ }^{*}<<0.05, T Q+S t r o k e ~ V s$. Stroke $\star \star P<0.01, T P+$ Stroke Vs. Stroke $P=n s$. All values are expressed as mean $+S E M, n=5$. 

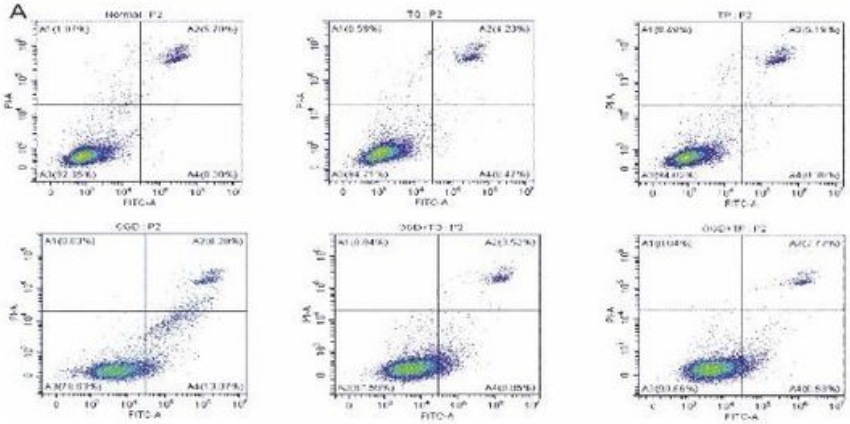

B
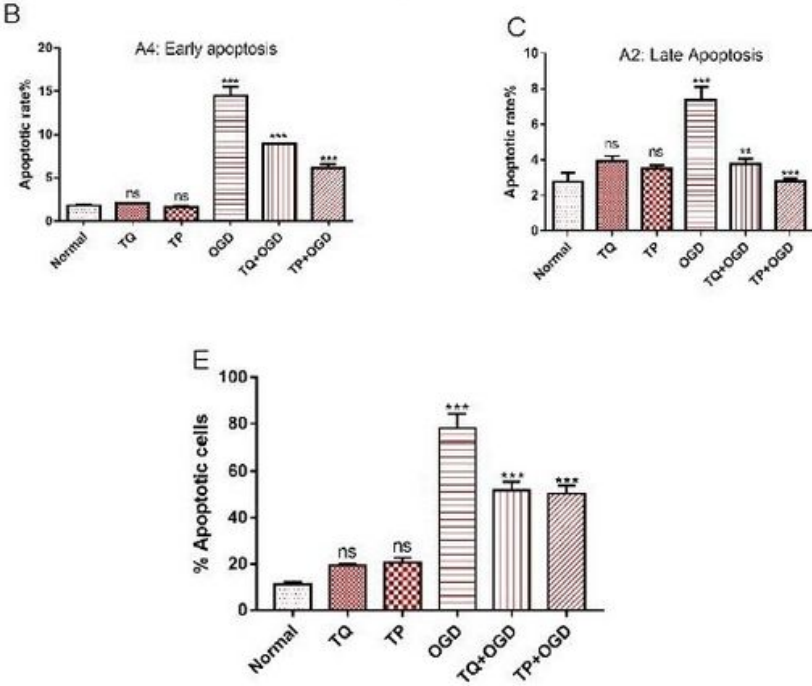
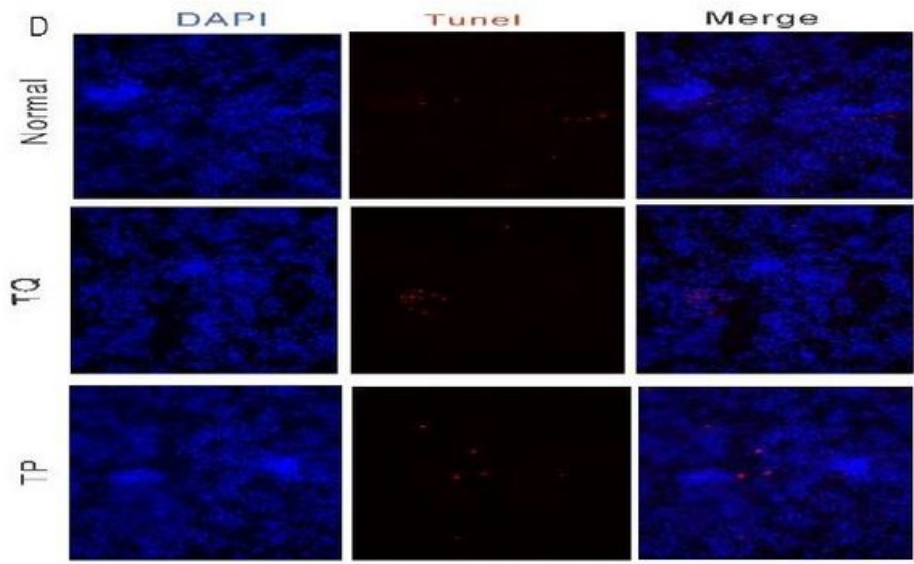

O
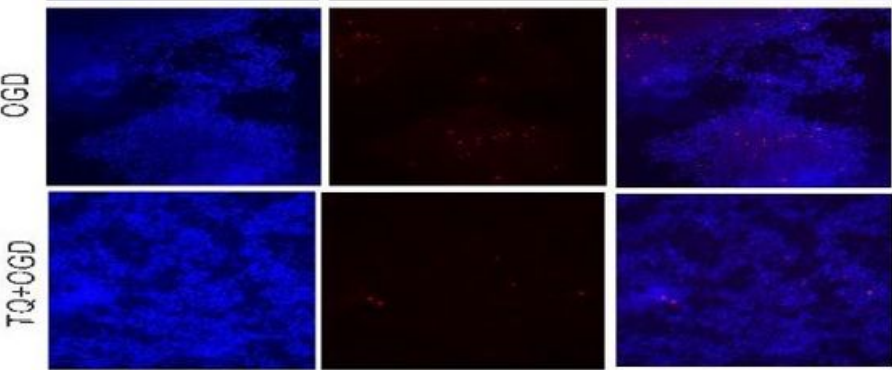

号
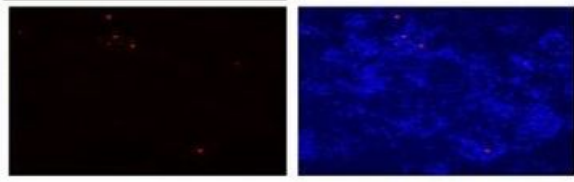

\section{Figure 9}

TQ therapy attenuates OGD/R induced apoptosis. (A) FACs analysis, (B) Early apoptosis; OGD Vs. Normal ***P<0.001, TQ@+ OGD Vs. OGD ***P<0.001 an TP+OGD Vs. OGD ***P<0.001.(C) Late apoptosis; OGD Vs. Normal ${ }^{* \star *} P<0.001, T Q+O G D$ Vs. OGD $* * P<0.01$ and TP+ OGD Vs. OGD $* \star * P<0.001$. (D) Tunel staining, (E) \% Apoptotic cells; OGD Vs. Normal ***P<0.001, TQ@+ OGD Vs. OGD ***P<Q.001 and TP+ OGD Vs. OGD ***P<0.001. 

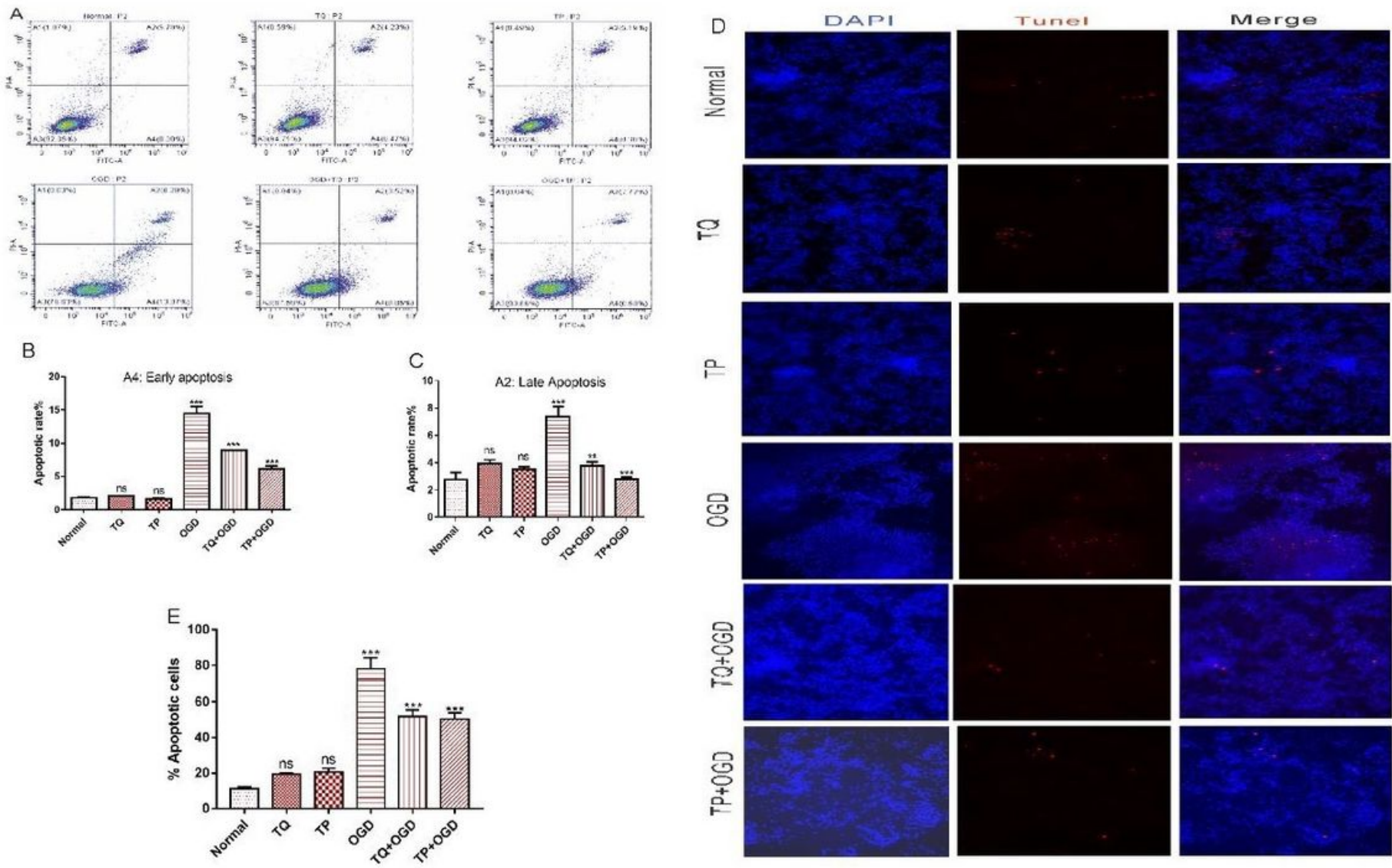

\section{Figure 9}

TQ therapy attenuates OGD/R induced apoptosis. (A) FACs analysis, (B) Early apoptosis; OGD Vs. Normal ***P<0.001, TQ@+ OGD Vs. OGD ***P<0.001 an TP+OGD Vs. OGD ***P<0.001. (C) Late apoptosis; OGD Vs. Normal ${ }^{\star *} \mathrm{P}<0.001, T Q+O G D$ Vs. OGD $* * P<0.01$ and TP+OGD Vs. OGD $* \star * P<0.001$. (D) Tunel

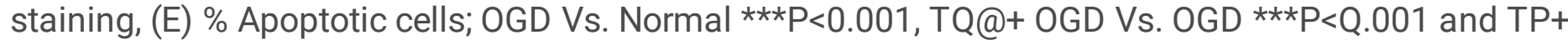
OGD Vs. OGD ***P<0.001. 

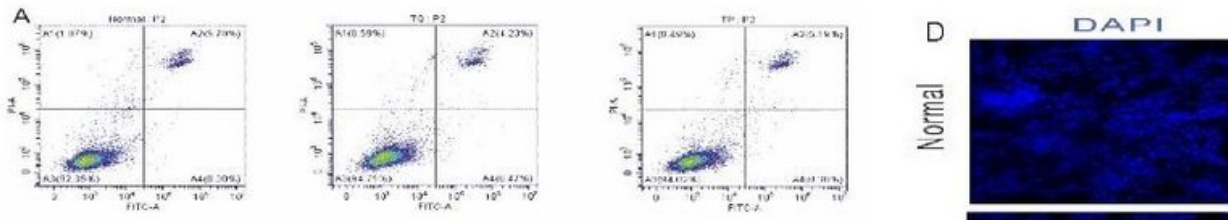

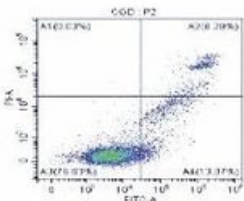

B
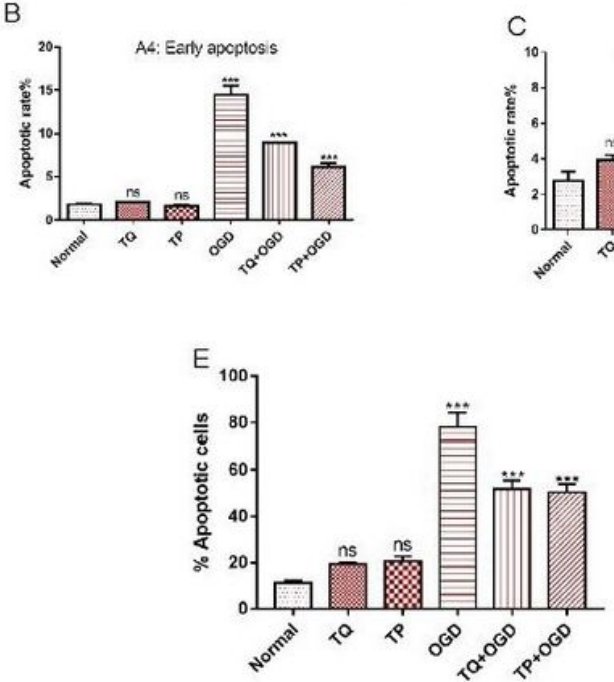
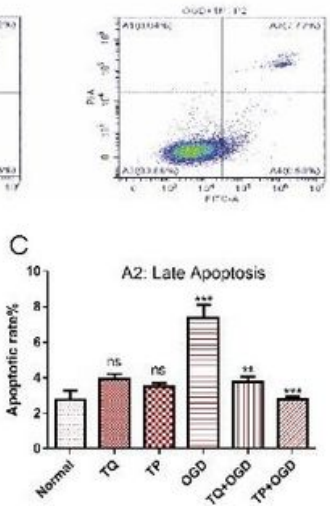

응
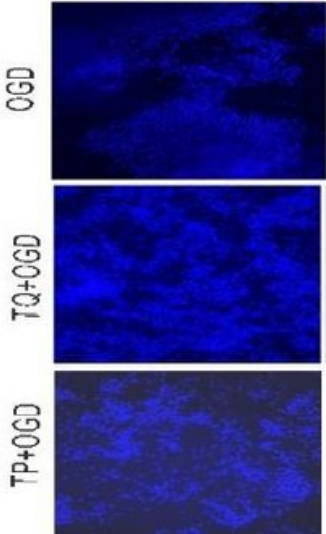

Merge
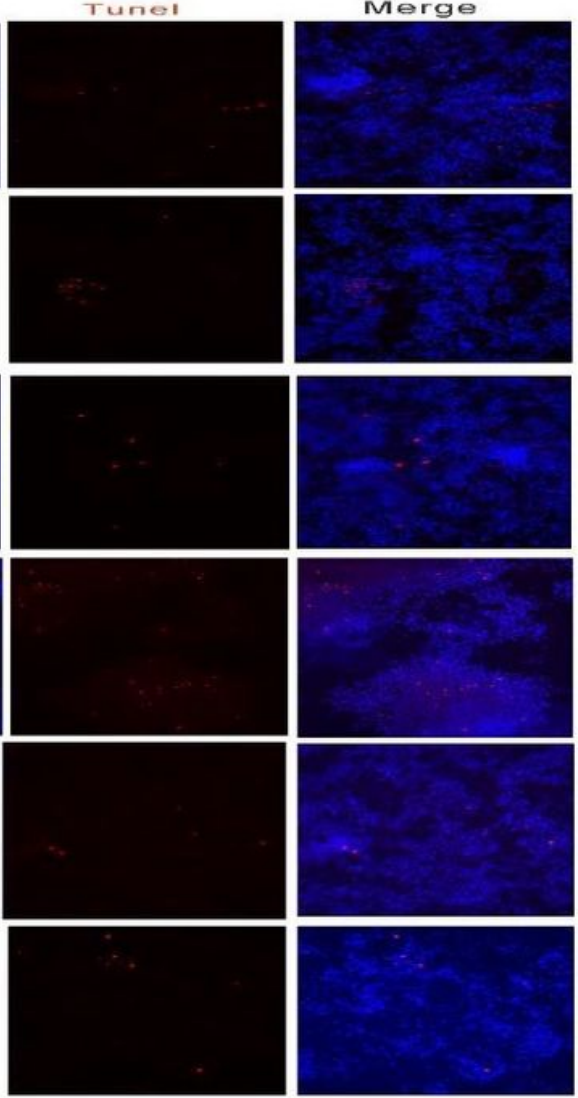

\section{Figure 9}

TQ therapy attenuates OGD/R induced apoptosis. (A) FACs analysis, (B) Early apoptosis; OGD Vs. Normal ***P<0.001, TQ@+ OGD Vs. OGD ***P<0.001 an TP+OGD Vs. OGD ***P<0.001. (C) Late apoptosis; OGD Vs. Normal ${ }^{\star *} \mathrm{P}<0.001, T Q+O G D$ Vs. OGD $* * P<0.01$ and TP+ OGD Vs. OGD $* \star * P<0.001$. (D) Tunel

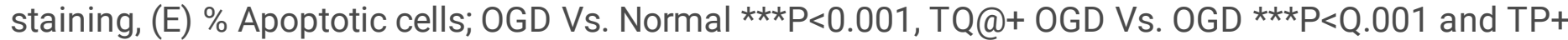
OGD Vs. OGD $* * * P<0.001$. 

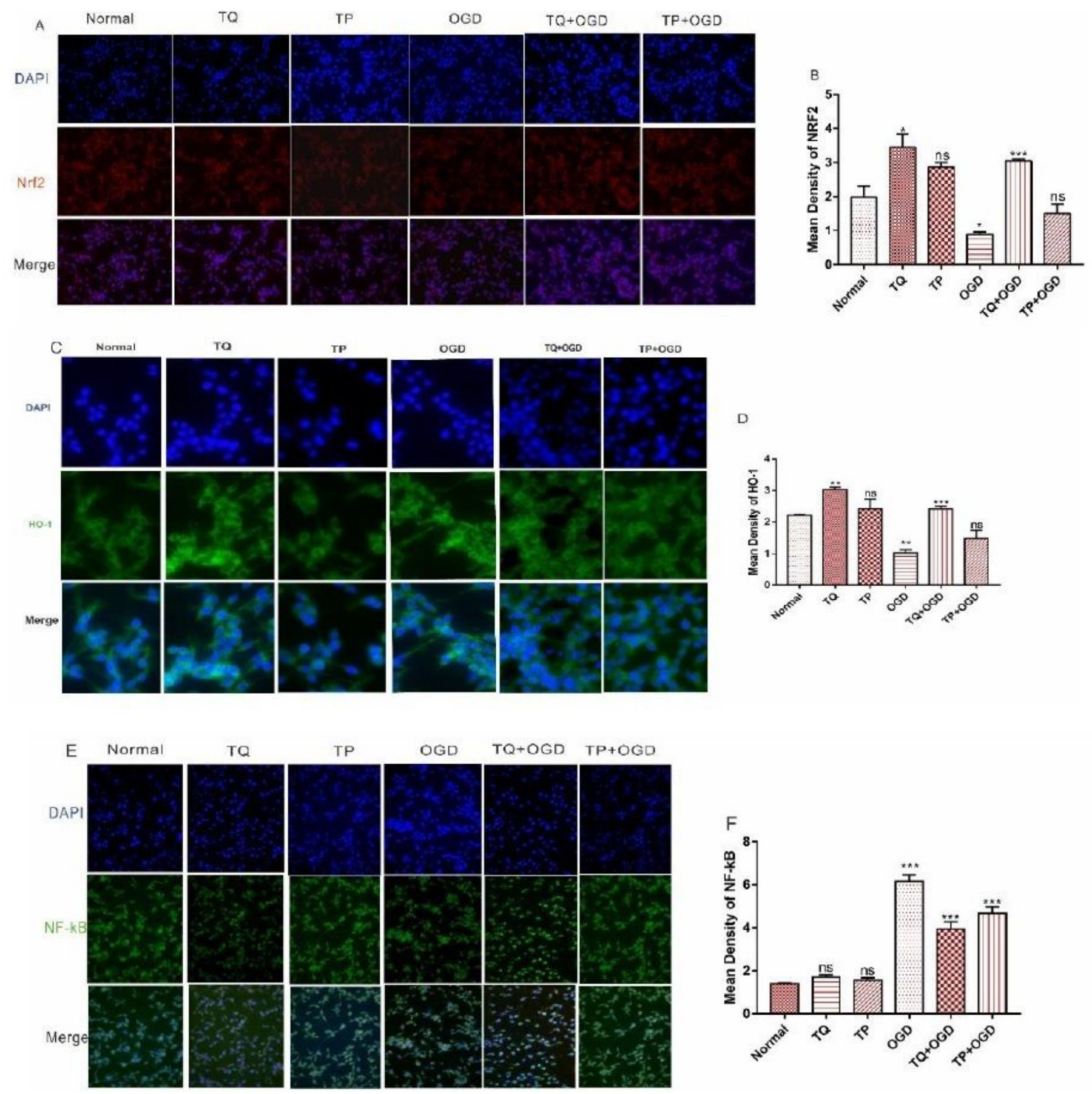

Figure 10

TQ therapy alleviates OGD/R-induced apoptosis via Inhibition of NF - KB and activation of Nrf2/HO -1 Pathway. A, Immunofluorescence staining of Nrf2. B, Mean density of Nrf2. C, Immunofluorescence staining of HO-1. D, mean density of HO -1. E, Immunofluorescence staining of NF -kB (F) mean density of $\mathrm{NF}-\mathrm{kB}$. All values are expressed as mean $+\mathrm{SEM}, \mathrm{n}=3$. 

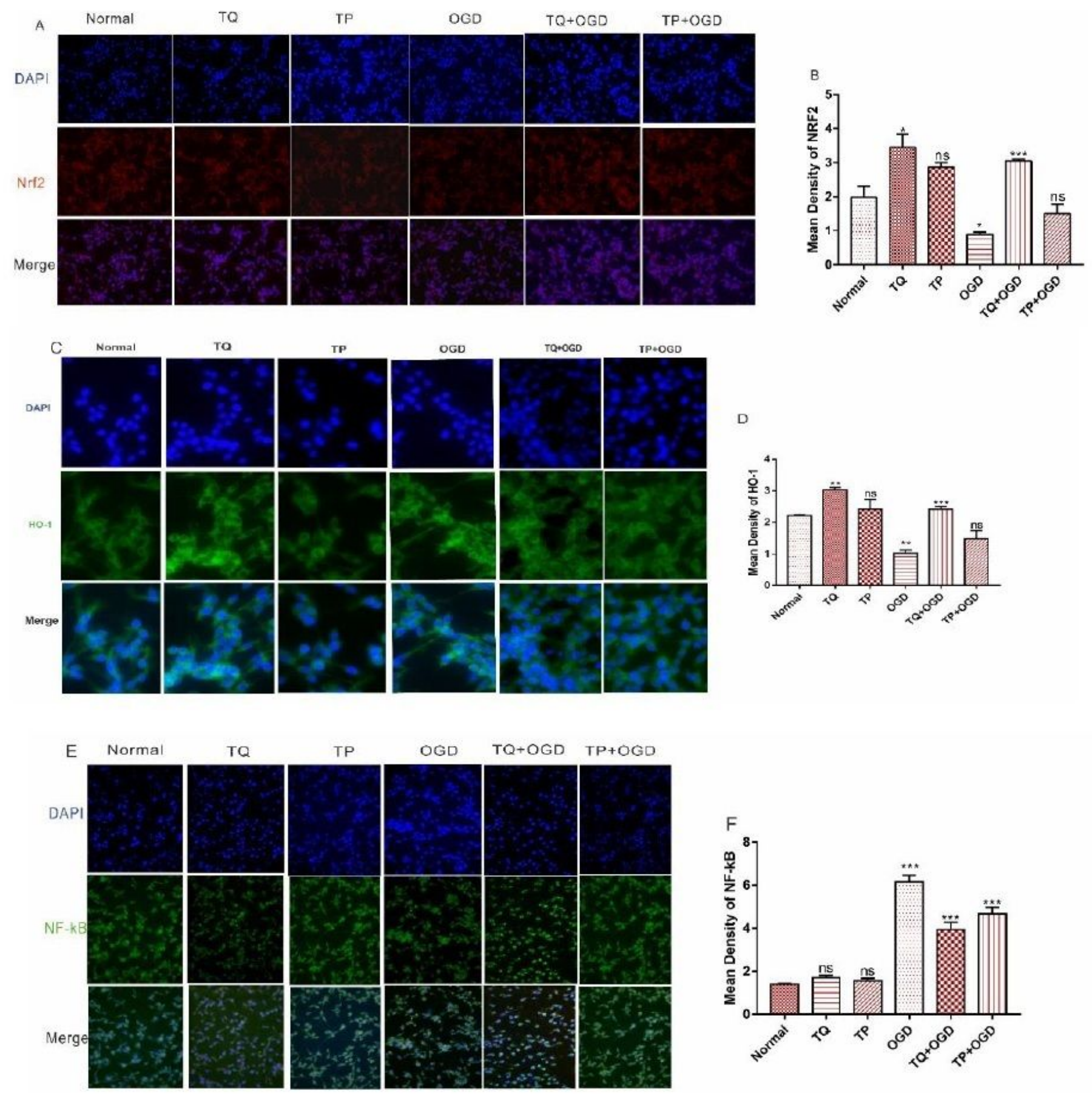

Figure 10

TQ therapy alleviates OGD/R-induced apoptosis via Inhibition of NF - KB and activation of Nrf2/HO -1 Pathway. A, Immunofluorescence staining of Nrf2. B, Mean density of Nrf2. C, Immunofluorescence staining of HO-1. D, mean density of HO -1. E, Immunofluorescence staining of NF -kB (F) mean density of $\mathrm{NF}-\mathrm{kB}$. All values are expressed as mean $+\mathrm{SEM}, \mathrm{n}=3$. 

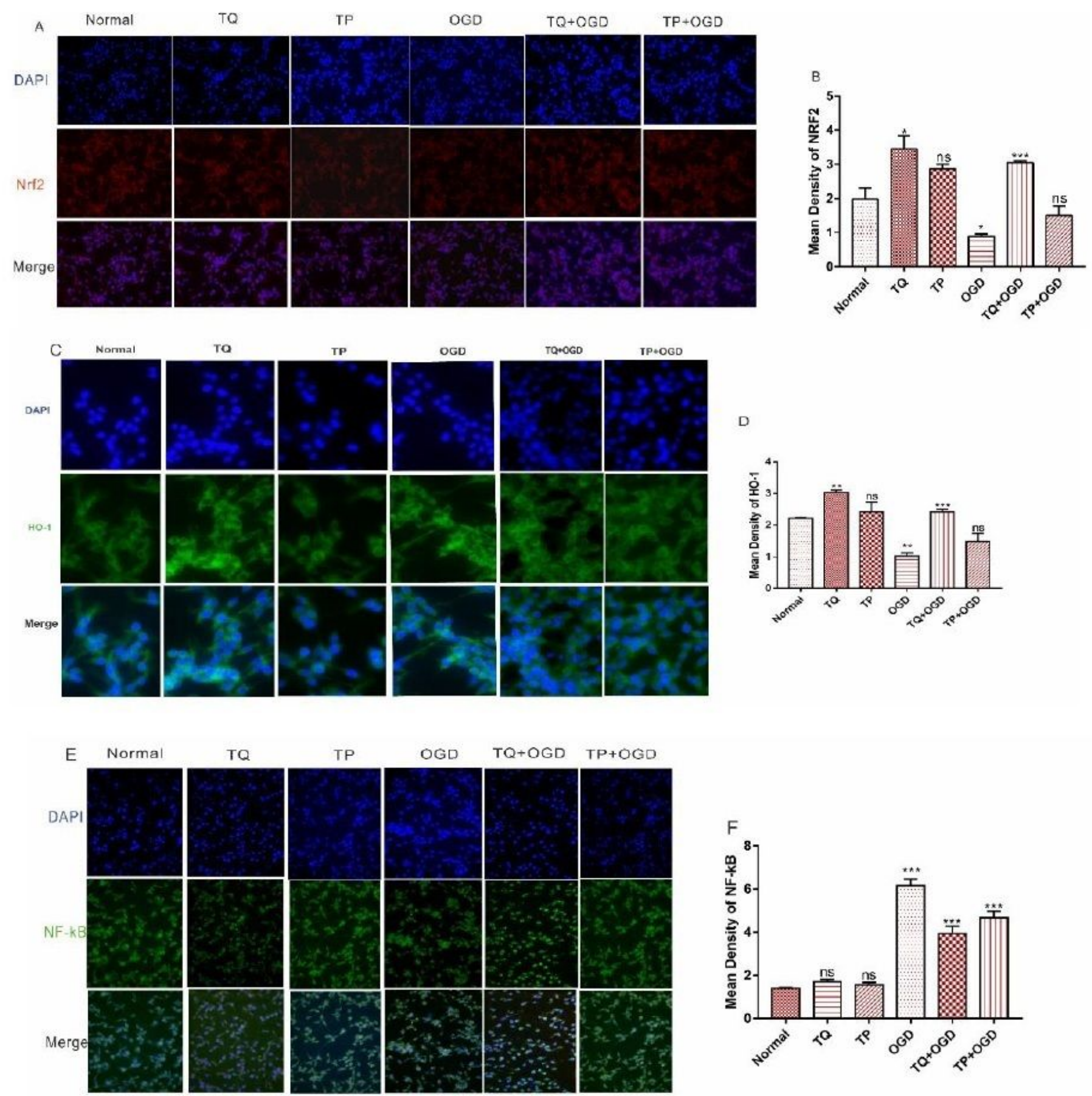

Figure 10

TQ therapy alleviates OGD/R-induced apoptosis via Inhibition of NF - KB and activation of Nrf2/HO -1 Pathway. A, Immunofluorescence staining of Nrf2. B, Mean density of Nrf2. C, Immunofluorescence staining of HO-1. D, mean density of HO -1. E, Immunofluorescence staining of NF -kB (F) mean density of $\mathrm{NF}-\mathrm{kB}$. All values are expressed as mean $+\mathrm{SEM}, \mathrm{n}=3$.

\section{Supplementary Files}

This is a list of supplementary files associated with this preprint. Click to download. 
- SupplementaryData.docx

- SupplementaryData.docx

- SupplementaryData.docx

- Graphicalabstract.pdf

- Graphicalabstract.pdf

- Graphicalabstract.pdf 